\title{
Measurements of the $E$-mode polarization and temperature- $E$-mode correlation of the CMB from SPT-3G 2018 data
}

D. Dutcher $\oplus_{1,}^{1,2}$ L. Balkenhol, ${ }^{3}$ P. A. R. Ade, ${ }^{4}$ Z. Ahmed ${ }^{5,6}$ E. Anderes, ${ }^{7}$ A. J. Anderson, ${ }^{8,2}$ M. Archipley, ${ }^{9}$ J. S. Avva, ${ }^{10}$ K. Aylor, ${ }^{11}$ P. S. Barry, ${ }^{12,2}$ R. Basu Thakur, ${ }^{2,13}$ K. Benabed, ${ }^{14}$ A. N. Bender, ${ }^{12,2}$ B. A. Benson, ${ }^{8,2,15}$ F. Bianchini, ${ }^{5,16,3}$ L. E. Bleem, ${ }^{12,2}$ F. R. Bouchet, ${ }^{14}$ L. Bryant, ${ }^{17}$ K. Byrum, ${ }^{12}$ J. E. Carlstrom, ${ }^{2,17,1,12,15}$ F. W. Carter, ${ }^{12,2}$ T. W. Cecil, ${ }^{12}$ C. L. Chang, ${ }^{12,2,15}$ P. Chaubal ${ }^{3}$ G. Chen, ${ }^{18}$ H.-M. Cho, ${ }^{6}$ T.-L. Chou, ${ }^{1,2}$ J.-F. Cliche, ${ }^{19}$ T. M. Crawford, ${ }^{2,15}$ A. Cukierman, ${ }^{5,6,16}$ C. Daley, ${ }^{9}$ T. de Haan, ${ }^{20}$ E. V. Denison, ${ }^{21}$ K. Dibert, ${ }^{152}$ J. Ding, ${ }^{22}$ M. A. Dobbs, ${ }^{19,23}$ W. Everett,${ }^{24}$ C. Feng, ${ }^{25}$

K. R. Ferguson, ${ }^{26}$ A. Foster, ${ }^{27}$ J. Fu, ${ }^{9}$ S. Galli, ${ }^{14}$ A. E. Gambrel, ${ }^{2}$ R. W. Gardner, ${ }^{17}$ N. Goeckner-Wald, ${ }^{16,5}$ R. Gualtieri, ${ }^{12}$ S. Guns, ${ }^{10}$ N. Gupta, ${ }^{3,28}$ R. Guyser, ${ }^{9}$ N. W. Halverson, ${ }^{24,29}$ A. H. Harke-Hosemann, ${ }^{12,9}$ N. L. Harrington, ${ }^{10}$

J. W. Henning, ${ }^{12,2}$ G. C. Hilton, ${ }^{21}$ E. Hivon, ${ }^{14}$ G. P. Holder, ${ }^{25}$ W. L. Holzapfel, ${ }^{10}$ J. C. Hood, ${ }^{2}$ D. Howe, ${ }^{18}$ N. Huang,${ }^{10}$ K. D. Irwin, ${ }^{5,16,6}$ O. B. Jeong, ${ }^{10}$ M. Jonas, ${ }^{8}$ A. Jones, ${ }^{18}$ T. S. Khaire, ${ }^{22}$ L. Knox, ${ }^{11}$ A. M. Kofman ${ }^{30}$ M. Korman ${ }^{27}$

D. L. Kubik, ${ }^{8}$ S. Kuhlmann, ${ }^{12}$ C.-L. Kuo, ${ }^{5,16,6}$ A. T. Lee, ${ }^{10,31}$ E. M. Leitch, ${ }^{2,15}$ A. E. Lowitz, ${ }^{2}$ C. Lu, ${ }^{25}$ S. S. Meyer, ${ }^{2,17,1,15}$ D. Michalik, ${ }^{18}$ M. Millea, ${ }^{10}$ J. Montgomery, ${ }^{19}$ A. Nadolski, ${ }^{9}$ T. Natoli, ${ }^{2}$ H. Nguyen, ${ }^{8}$ G. I. Noble, ${ }^{19}$ V. Novosad, ${ }^{22}$ Y. Omori, ${ }^{5,16}$ S. Padin, ${ }^{2,13}$ Z. Pan, ${ }^{12,2,1}$ P. Paschos, ${ }^{17}$ J. Pearson, ${ }^{22}$ C. M. Posada, ${ }^{22}$ K. Prabhu, ${ }^{11}$ W. Quan, ${ }^{1,2}$

S. Raghunathan, ${ }^{32,26}$ A. Rahlin, ${ }^{8,2}$ C. L. Reichardt, ${ }^{3}$ D. Riebel, ${ }^{18}$ B. Riedel, ${ }^{17}$ M. Rouble, ${ }^{19}$ J. E. Ruhl, ${ }^{27}$ J. T. Sayre, ${ }^{24}$ E. Schiappucci, ${ }^{3}$ E. Shirokoff, ${ }^{2,15}$ G. Smecher, ${ }^{33}$ J. A. Sobrin, ${ }^{1,2}$ A. A. Stark, ${ }^{34}$ J. Stephen, ${ }^{17}$ K. T. Story, ${ }^{5,16}$ A. Suzuki, ${ }^{31}$ K. L. Thompson, ${ }^{5,16,6}$ B. Thorne ${ }^{11}$ C. Tucker, ${ }^{4}$ C. Umilta, ${ }^{25}$ L. R. Vale, ${ }^{21}$ K. Vanderlinde, ${ }^{35,36}$ J. D. Vieira, ${ }^{9,25}$ G. Wang, ${ }^{12}$ N. Whitehorn, ${ }^{32,26}$ W. L. K. Wu, ${ }^{5,6}$ V. Yefremenko, ${ }^{12}$ K. W. Yoon, ${ }^{5,16,6}$ and M. R. Young ${ }^{36}$

(SPT-3G Collaboration)

\footnotetext{
${ }^{1}$ Department of Physics, University of Chicago, 5640 South Ellis Avenue, Chicago, Illinois 60637, USA

${ }^{2}$ Kavli Institute for Cosmological Physics, University of Chicago, 5640 South Ellis Avenue, Chicago, Illinois 60637, USA

${ }^{3}$ School of Physics, University of Melbourne, Parkville, Victoria 3010, Australia

${ }^{4}$ School of Physics and Astronomy, Cardiff University, Cardiff CF24 3YB, United Kingdom

${ }^{5}$ Kavli Institute for Particle Astrophysics and Cosmology, Stanford University, 452 Lomita Mall, Stanford, California 94305, USA

${ }^{6}$ SLAC National Accelerator Laboratory, 2575 Sand Hill Road, Menlo Park, California 94025, USA

${ }^{7}$ Department of Statistics, University of California, One Shields Avenue, Davis, California 95616, USA

${ }^{8}$ Fermi National Accelerator Laboratory, MS209, P.O. Box 500, Batavia, Illinois 60510, USA

${ }^{9}$ Department of Astronomy, University of Illinois Urbana-Champaign, 1002 West Green Street, Urbana, Illinois 61801, USA

${ }^{10}$ Department of Physics, University of California, Berkeley, California 94720, USA

${ }^{11}$ Department of Physics \& Astronomy, University of California, One Shields Avenue, Davis, California 95616, USA

${ }^{12}$ High-Energy Physics Division, Argonne National Laboratory, 9700 South Cass Avenue., Argonne, Illinois 60439, USA

${ }^{13}$ California Institute of Technology, 1200 East California Boulevard., Pasadena, California 91125, USA

${ }^{14}$ Institut d'Astrophysique de Paris, UMR 7095, CNRS \& Sorbonne Université, 98 bis boulevard Arago, 75014 Paris, France

${ }^{15}$ Department of Astronomy and Astrophysics, University of Chicago, 5640 South Ellis Avenue, Chicago, Illinois 60637, USA

${ }^{16}$ Department of Physics, Stanford University, 382 Via Pueblo Mall, Stanford, California 94305, USA

${ }^{17}$ Enrico Fermi Institute, University of Chicago, 5640 South Ellis Avenue, Chicago, Illinois 60637, USA

${ }^{18}$ University of Chicago, 5640 South Ellis Avenue, Chicago, Illinois 60637, USA

${ }^{19}$ Department of Physics and McGill Space Institute, McGill University, 3600 Rue University, Montreal, Quebec H3A 2T8, Canada

${ }^{20}$ High Energy Accelerator Research Organization (KEK), Tsukuba, Ibaraki 305-0801, Japan

${ }^{21}$ NIST Quantum Devices Group, 325 Broadway Mailcode 817.03, Boulder, Colorado 80305, USA

${ }^{22}$ Materials Sciences Division, Argonne National Laboratory, 9700 South Cass Avenue, Argonne, Illinois 60439, USA

${ }^{23}$ Canadian Institute for Advanced Research, CIFAR Program in Gravity and the Extreme Universe,
} Toronto, Ontario M5G 1Z8, Canada 


\author{
${ }^{24}$ CASA, Department of Astrophysical and Planetary Sciences, \\ University of Colorado, Boulder, Colorado 80309, USA \\ ${ }^{25}$ Department of Physics, University of Illinois Urbana-Champaign, \\ 1110 West Green Street, Urbana, Illinois 61801, USA \\ ${ }^{26}$ Department of Physics and Astronomy, University of California, Los Angeles, California 90095, USA \\ ${ }^{27}$ Department of Physics, Case Western Reserve University, Cleveland, Ohio 44106, USA \\ ${ }^{28}$ CSIRO Astronomy and Space Science, PO Box 1130, Bentley Western Australia 6102, Australia \\ ${ }^{29}$ Department of Physics, University of Colorado, Boulder, Colorado 80309, USA \\ ${ }^{30}$ Department of Physics \& Astronomy, University of Pennsylvania, \\ 209 S. 33rd Street, Philadelphia Pennsylvania 19064, USA \\ ${ }^{31}$ Physics Division, Lawrence Berkeley National Laboratory, Berkeley, California 94720, USA \\ ${ }^{32}$ Department of Physics and Astronomy, Michigan State University, East Lansing, Michigan 48824, USA \\ ${ }^{33}$ Three-Speed Logic, Inc., Victoria, British Columbia V8S 3Z5, Canada \\ ${ }^{34}$ Harvard-Smithsonian Center for Astrophysics, 60 Garden Street, Cambridge, \\ Massachusetts 02138, USA \\ ${ }^{35}$ Dunlap Institute for Astronomy \& Astrophysics, University of Toronto, \\ 50 St. George Street, Toronto, Ontario M5S 3H4, Canada \\ ${ }^{36}$ David A. Dunlap Department of Astronomy \& Astrophysics, University of Toronto, \\ 50 St. George Street, Toronto, Ontario M5S 3H4, Canada
}

(Received 7 January 2021; accepted 4 June 2021; published 13 July 2021)

\begin{abstract}
We present measurements of the $E$-mode $(E E)$ polarization power spectrum and temperature- $E$-mode $(T E)$ cross-power spectrum of the cosmic microwave background using data collected by SPT-3G, the latest instrument installed on the South Pole Telescope. This analysis uses observations of a $1500 \mathrm{deg}^{2}$ region at 95, 150, and $220 \mathrm{GHz}$ taken over a four-month period in 2018 . We report binned values of the $E E$ and $T E$ power spectra over the angular multipole range $300 \leq \ell<3000$, using the multifrequency data to construct six semi-independent estimates of each power spectrum and their minimum-variance combination. These measurements improve upon the previous results of SPTpol across the multipole ranges $300 \leq \ell \leq 1400$ for $E E$ and $300 \leq \ell \leq 1700$ for $T E$, resulting in constraints on cosmological parameters comparable to those from other current leading ground-based experiments. We find that the SPT-3G data set is well fit by a $\Lambda \mathrm{CDM}$ cosmological model with parameter constraints consistent with those from Planck and SPTpol data. From SPT-3G data alone, we find $H_{0}=68.8 \pm 1.5 \mathrm{~km} \mathrm{~s}^{-1} \mathrm{Mpc}^{-1}$ and $\sigma_{8}=0.789 \pm 0.016$, with a gravitational lensing amplitude consistent with the $\Lambda$ CDM prediction $\left(A_{L}=0.98 \pm 0.12\right)$. We combine the SPT-3G and the Planck data sets and obtain joint constraints on the $\Lambda$ CDM model. The volume of the $68 \%$ confidence region in six-dimensional $\Lambda$ CDM parameter space is reduced by a factor of 1.5 compared to Planck-only constraints, with no significant shifts in central values. We note that the results presented here are obtained from data collected during just half of a typical observing season with only part of the focal plane operable, and that the active detector count has since nearly doubled for observations made with SPT-3G after 2018.
\end{abstract}

DOI: $10.1103 /$ PhysRevD.104.022003

\section{INTRODUCTION}

The cosmic microwave background (CMB) is a rich source of information about the early Universe and its evolution over cosmic time. Density fluctuations present during the epoch of baryon-photon decoupling at $z \sim 1100$ imprint a faint temperature anisotropy on the $\mathrm{CMB}$, and measurements of the angular power spectrum of these anisotropies are a pillar of the standard six-parameter $\Lambda \mathrm{CDM}$ cosmological model. Satellite measurements of the CMB temperature power spectrum are now cosmic variance limited from the largest angular scales down to roughly seven arcminutes [1] (corresponding to angular multipoles $\ell \lesssim 1600$ ), and ground-based observations extend these measurements to arcminute scales, at which point other sources of millimeter-wave anisotropy, including the cosmic infrared background, radio galaxies, and the thermal and kinetic Sunyaev-Zel'dovich (SZ) effects, begin to dominate over the primary $\mathrm{CMB}$ temperature signal. $[2,3]$.

The CMB anisotropies are linearly polarized at the $10 \%$ level as a result of local quadrupole fluctuations at the surface of last scattering [4]. The linear polarization map can be decomposed into two components: even-parity, curl-free " $E$-modes" and odd-parity, divergence-free " $B$-modes." Density fluctuations in the early Universe only create $E$-mode $\mathrm{CMB}$ polarization (to first order in the 
density contrast), while $B$-modes in the $\mathrm{CMB}$ can be created by tensor perturbations, such as primordial gravitational waves, or gravitational lensing of the $E$-modes by intervening large-scale structure [5-7]. In this paper we focus on the brighter $E$-mode component of this polarization. The $E$-mode $(E E)$ polarization power spectrum and the temperature- $E$-mode $(T E)$ cross-power spectrum can provide tighter constraints on cosmological parameters than temperature data alone [8], and they can be measured out to smaller angular scales on account of the low fractional polarization of extragalactic sources [9-11], providing a powerful consistency check of $\Lambda \mathrm{CDM}$.

The CMB temperature and polarization power spectra have been measured over a wide range of angular scales by the Planck satellite [1] and ground-based telescopes including the Atacama Cosmology Telescope (ACT) [12], BICEP/Keck [13], POLARBEAR [14,15], and the South Pole Telescope (SPT) (Ref. [16], hereafter H18) [17]. Several current and upcoming experiments aim to improve existing power spectrum constraints, including Advanced ACT [18], BICEP3/BICEP Array [19,20], POLARBEAR2/Simons Array [21], the Simons Observatory [22], and SPT-3G [23].

While the data are generally well described by $\Lambda \mathrm{CDM}$, there are mild tensions in parameter constraints between small and large angular scales [H18, 24,25] and significant tensions between CMB measurements and late-time cosmological probes, most notably in the value of the Hubble constant $H_{0}$ [26,27]. Upcoming measurements of the high$\ell \mathrm{CMB}$ power spectra may shed light on the origin of these tensions.

In this paper, we present the first science results from SPT$3 \mathrm{G}$, the latest survey instrument installed on the South Pole Telescope [28]. We report measurements of the $E E$ and $T E$ power spectra over the angular multipole range $300 \leq \ell<$ 3000 from observations of a $\sim 1500 \mathrm{deg}^{2}$ region undertaken during a four-month period of 2018, and we present the resulting constraints on cosmological parameters.

The shortened 2018 observing season is the result of telescope downtime at the beginning of the year due to an issue with the telescope drive system, which caused damage to detector readout and rendered approximately half the focal plane inoperable. We addressed the issue at the close of 2018 and have since seen normal performance during the 2019 and 2020 observing seasons. Nevertheless, the data collected during 2018 is already sufficient to provide the most sensitive measurements made to date with SPT over the multipole ranges $300 \leq \ell \leq 1400$ for $E E$ and $300 \leq \ell \leq 1700$ for $T E$. The resulting constraints on cosmological parameters from the SPT-3G 2018 power spectra improve upon those set by SPTpol [H18] and are competitive with those from other current leading groundbased experiments [29].

This paper is organized as follows. We begin with an overview of the SPT-3G instrument in Sec. II. In Sec. III we discuss the scanning strategy of the telescope, low-level data processing, and the coadded maps. In Sec. IV we detail the absolute calibration of the maps and the procedure used for obtaining unbiased measurements of power spectra. Tests for systematic error in the data collection or processing steps are discussed in Sec. V. The method for obtaining constraints on cosmological parameters from the power spectra measurements is detailed in Sec. VI. We present final band-power measurements in Sec. VII and discuss the resulting constraints on cosmological parameters in Sec. VIII.

\section{THE SPT-3G INSTRUMENT}

Deployed in early 2017, SPT-3G is the third survey camera to be installed on SPT. SPT-3G is a significant upgrade over the previous instruments, utilizing redesigned wide-field optics to increase the field of view from $\sim 1 \mathrm{deg}^{2}$ to $2.8 \mathrm{deg}^{2}$ and populating the $3.5 \times$ larger focal plane area with multichroic pixels. Light rays from the $10 \mathrm{~m}$ primary mirror are redirected by a $2 \mathrm{~m}$ ellipsoidal secondary mirror and $1 \mathrm{~m}$ flat tertiary mirror into the receiver cryostat [30], in which three $0.72 \mathrm{~m}$ diameter anti-reflection-coated alumina lenses [31] reimage the Gregorian focus onto the detectors. The SPT-3G receiver can be divided functionally into two cryostats that share a common vacuum: an optics cryostat that contains the cold optical elements, and a detector cryostat that contains the detectors and associated readout electronics. Each cryostat is cooled to $4 \mathrm{~K}$ by its own dedicated pulse tube cooler, and the detectors are further cooled to their operating temperature of $300 \mathrm{mK}$ by a custom closed-cycle three-stage helium sorption refrigerator manufactured by Chase Research Cryogenics. ${ }^{1}$ With the cooling power required by the SPT-3G instrument, the refrigerator can provide a stable base temperature of $300 \mathrm{mK}$ for approximately 17 hours before it must be raised to $4 \mathrm{~K}$ for a 4.5 hour recharge cycle.

The $0.43 \mathrm{~m}$ diameter focal plane is populated with $\sim 16000$ transition-edge sensor (TES) bolometers fabricated on ten monolithic $150 \mathrm{~mm}$ silicon wafers. Each detector wafer contains an array of 269 multichroic dual linearly polarized pixels, with each pixel consisting of a broadband sinuous antenna coupled to TES bolometers via superconducting microstrip and in-line band-defining filters. This pixel architecture was originally developed for POLARBEAR-2/Simons Array [21] and is also planned for use by the Simons Observatory [32] and LiteBIRD [33] experiments. The SPT-3G pixels have three observing frequency bands, centered at 95, 150, and $220 \mathrm{GHz}$, and use six TES bolometers in each pixel to measure both polarization orientations in each band. Details of the SPT-3G detector wafer fabrication can be found in Refs. [34,35] and characterization of the 2018 deployed array in Ref. [36]. The detectors are read out using a $68 \times$

\footnotetext{
${ }^{1}$ http://www.chasecryogenics.com/.
} 
frequency-domain multiplexing system jointly developed by the SPT-3G and POLARBEAR-2 collaborations [37,38].

\section{OBSERVATIONS AND DATA REDUCTION}

\section{A. Observations}

The main SPT-3G survey field is a $\sim 1500 \mathrm{deg}^{2}$ region extending from $-42^{\circ}$ to $-70^{\circ}$ declination and from $20^{\mathrm{h}} 40^{\mathrm{m}} 0^{\mathrm{s}}$ to $3^{\mathrm{h}} 20^{\mathrm{m}} 0^{\mathrm{s}}$ right ascension, illustrated in Fig. 1. This survey footprint also overlaps the regions observed by the BICEP/Keck series of experiments $[13,20]$. We observe the full $1500 \mathrm{deg}^{2}$ via four $7.5^{\circ}$-tall subfields centered at $-44.75^{\circ},-52.25^{\circ},-59.75^{\circ}$, and $-67.25^{\circ}$ declination, respectively, with each subfield covering the full RA range. These subfields are chosen so as to maximize telescope scanning efficiency while minimizing fluctuations in detector gain due to changes in atmospheric loading over the course of an observation.

The telescope observes each subfield in a raster pattern, performing constant-elevation sweeps in azimuth before making a small step in elevation and repeating. ${ }^{2}$ Each sweep of the telescope across the field, referred to as a scan, is performed at a constant $1 \mathrm{deg} / \mathrm{s}$ as measured on the azimuth bearing and takes approximately 100 seconds to cover the full azimuth range. The telescope performs one right-going scan and one left-going scan at each elevation step. A full subfield observation requires approximately 2.5 hours to complete, and two subfields are each observed three times during one observing day, defined by the combined fridge hold and cycle time. As the survey field is constantly above the horizon at the South Pole, the start of the observing day is allowed to drift with respect to sidereal time with no penalty to observing efficiency.

\section{B. Relative calibration}

We regularly conduct a series of calibration observations in order to relate the input power on each detector to $\mathrm{CMB}$ fluctuation temperature. This conversion is derived from observations of two Galactic HII regions that serve as relatively compact sources of mm-wave flux, RCW38 and MAT5a (NGC 3576). RCW38 is located at RA: $8^{\mathrm{h}} 59^{\mathrm{m}} 5^{\mathrm{s}}$ Dec: $-47^{\circ} 30^{\prime} 36^{\prime \prime}$ and is used for the two higher-declination fields, while MAT5a is located at RA: $11^{\mathrm{h}} 11^{\mathrm{m}} 53^{\mathrm{s}}$ Dec: $-61^{\circ} 18^{\prime} 47^{\prime \prime}$ and is used for the two lower-declination fields. Dense scans are taken such that each pixel in the focal plane can form a complete map of the source; these per-detector maps are then compared to calibrated maps of RCW38 or MAT5a made by the SPT-SZ experiment. During 2018, such observations of either RCW38 or

\footnotetext{
${ }^{2}$ As a result of the telescope's unique location at the geographic South Pole, there is nearly a direct correspondence between the local coordinates of azimuth and elevation and the celestial coordinates of right ascension and (negative) declination, respectively.
}

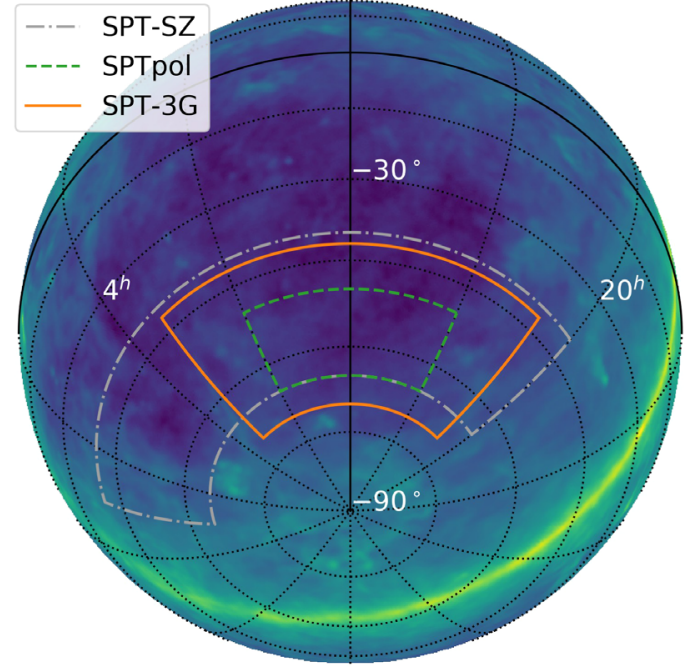

FIG. 1. The SPT-3G $1500 \mathrm{deg}^{2}$ survey field (orange, solid) overlaid on a Planck map of thermal dust emission [39]. Also shown are the SPTpol $500 \mathrm{deg}^{2}$ field [H18] (green, dashed) and the SPT-SZ $2500 \mathrm{deg}^{2}$ field [40] (gray, dot-dashed).

MAT5a were nominally performed once per observing day, depending on the pair of subfields to be observed, though in later seasons the cadence has been relaxed to one dense observation per HII region per week.

Temporal calibration shifts on shorter time scales are tracked using detector response to an internal calibration source ("the calibrator") and much shorter ( 10-minute) observations of the HII regions conducted before and after each $\mathrm{CMB}$ subfield observation. The short HII region observations also serve to monitor changes in atmospheric opacity. This procedure yields a conversion from input power to CMB fluctuation temperature for every detector and every observation, subject to statistical variations in the calibration observations and differences in beam shapes and passbands between SPT-3G and SPT-SZ. We expect these differences to bias the absolute calibration by less than $10 \%$, and we correct for this bias by comparing fully coadded maps to Planck (see Sec. IV F).

\section{TOD processing}

We apply a series of linear processing steps to the detector time-ordered data (TOD) to decrease and flatten the noise in the signal range, which in this analysis corresponds to approximately $0.3-6 \mathrm{~Hz}$. To reduce computing requirements, SPT-3G data is stored in a custom streaming file format ${ }^{3}$ that enables the data from only one scan of the telescope to be loaded into memory at once, and all TOD processing steps are performed on a scan-by-scan basis. Only data taken during the constant-velocity portion of each scan is used, and the data taken while the telescope is changing direction is discarded.

\footnotetext{
${ }^{3}$ https://github.com/CMB-S4/spt3g_software.
} 
The TOD used in this analysis have a sample rate of $76.3 \mathrm{~Hz}$, which while already downsampled by a factor of 2 from the native sample rate of $152.6 \mathrm{~Hz}$, is still faster than required to measure the angular scales of interest here. To prevent high-frequency noise from aliasing down into the signal band when binning data into map pixels, we apply a Fourier-space filter with functional form $e^{\left(-\ell_{x} / \ell_{0}\right)^{6}}$ and lowpass cutoff $\ell_{0}=6600$. The relation between $\ell_{x}$ and temporal frequency is determined by on-sky scanning speed and is recomputed for each scan of the telescope; at the center of the field, $\ell=6600$ corresponds to approximately $10 \mathrm{~Hz}$.

We also high-pass filter the data to remove the effects of slow signals, such as those caused by atmospheric noise or thermal drifts of the detector cold stage. To do this, we first fit and subtract up to a 19th-order Legendre polynomial from the TOD before projecting out Fourier modes corresponding to angular scales below $\ell_{x}=300$. The polynomial subtraction serves to remove lower-order modes that are not well described by Fourier decomposition, e.g., a linear slope. During this filtering step, TOD samples in which a detector was pointed within $5^{\prime}$ of a point source brighter than $50 \mathrm{mJy}$ at $150 \mathrm{GHz}$ are masked in that detector's TOD to prevent filter-induced ringing artifacts in the output map.

We apply one additional filtering step, referred to as the common-mode $(\mathrm{CM})$ filter, in which the signals from detectors in a specified group are averaged together, and the result is then subtracted from each of those detectors' TOD, thereby removing any common signal. Here we use all detectors in the same frequency band on the same detector wafer to form the common mode, averaging across polarization orientations. This effectively imposes a highpass filter that removes most of the temperature signal on scales larger than the angular extent of a wafer $(\ell \sim 500)$ while largely preserving the polarization signal. The TOD samples corresponding to point sources brighter than $50 \mathrm{mJy}$ at $150 \mathrm{GHz}$ are interpolated over during the CM filter to avoid creating spurious decrements in the map.

\section{Data quality cuts}

To prevent low-quality data from degrading a map, detectors with abnormal behavior or properties are flagged on a per-scan basis during TOD processing. If a detector is flagged, its data is dropped from the corresponding scan. Some of the lower-level reasons to flag a detector include a failure to properly bias or entering a fully superconducting state during an observation, poor calibration data due to noise fluctuations or detector operational issues, and readout errors during data acquisition. An average of 448 detectors are flagged in each scan for such reasons. We also flag detectors for irregular TOD features, on average removing an additional 342 detectors per scan due to 1) abrupt, large deviations from a rolling average, or "glitches," with causes including cosmic-ray hits and vibrations within the cryostat, or 2) excess line power in the $8-10 \mathrm{~Hz}$ range, thought to originate from instability in the detector or readout circuit.

In addition to the cuts above, we do not include one of the detector wafers in this analysis, as its TOD are dominated by a series of noise lines at multiples of 1.0 and $1.4 \mathrm{~Hz}$, the latter of which corresponds to the frequency of the pulse-tube cooler used in the cryostat. This wafer has been replaced for subsequent observing seasons.

After filtering, an inverse-variance weight $w_{i}$ is computed for each detector based on the noise in its TOD from 1-4 Hz. The distribution of weights is examined for outliers, and detectors with weights three sigma above or below the mean are flagged, removing on average another 33 detectors from each scan. The map for a given observation is constructed as a weighted average of the data from all detectors (after filtering and cuts) using this weight distribution.

Beyond cuts on individual detectors, whole scans are dropped from the observation data if there are errors in the telescope pointing information or if fewer than $\sim 50 \%$ of active bolometers pass cuts. Entire observations are cut if there was an error with data acquisition, if all detectors were flagged (e.g., due to a failed calibration observation), or if the helium in the sorption refrigerator ran out during the observation. After cutting 17 such observations, there are 562 subfield observations remaining, with an approximate average of 6600 active detectors equally distributed among the three frequency bands per observation.

\section{E. Maps}

We use the same map-making methodology as implemented for SPTpol analyses $[16,17,41,42]$ and described in Ref. [43], here binning the TOD into $2^{\prime}$ square pixels using the Lambert azimuthal equal-area projection.

The full-season coadded maps of temperature, Stokes $Q$, and Stokes $U$ for $150 \mathrm{GHz}$ are shown in Fig. 2. The crosshatched patterns in the $Q$ and $U$ polarization maps are indicative of measuring $E$-modes at high signal-to-noise. The $E$-mode polarization map itself is shown in Fig. 3. The noise levels in the coadded maps are measured by differencing two half-depth coadded maps and calculating the power spectrum of the result, correcting for the transfer function effects of the TOD filtering described above. The map depths as a function of $\ell$ for both temperature and polarization data are shown in Fig. 4; averaged over the range $1000<\ell<2000$, the polarized map depths at 95, 150 , and $220 \mathrm{GHz}$ are $29.6,21.2$, and $75 \mu \mathrm{K}$-arcmin, respectively.

From the 562 subfield observations, we construct subsets of partial-depth full-field maps, or "bundles," that are then used as the basic inputs to the rest of the analysis. The bundles are constructed by chronologically coadding observations within each subfield until the combined unpolarized weight approaches $1 /\left(N_{\text {bundles }}\right)^{\text {th }}$ of the 


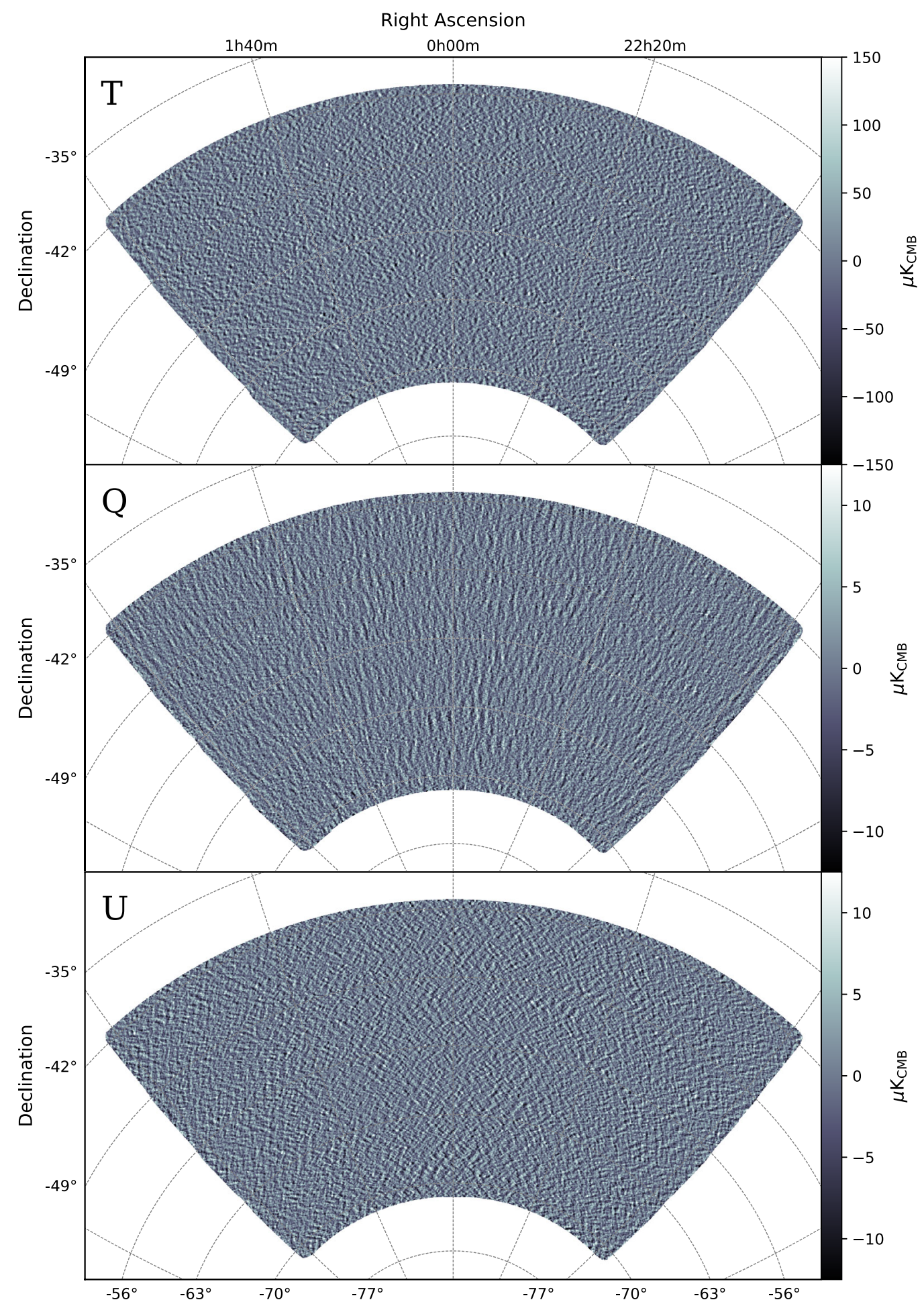

FIG. 2. SPT-3G $2018150 \mathrm{GHz}$ temperature (top), Stokes $Q$ (middle), and Stokes $U$ (bottom) maps. Note the factor of 10 difference in color scale between temperature and polarization maps. The data have been filtered to remove features larger than $\sim 0.5^{\circ}$, and the polarization maps have been smoothed by a $6^{\prime}$ FWHM Gaussian.

unpolarized weight in the full-season coadd, typically requiring 3-5 observations. The coadds from each of the four subfields are then combined to create one fullfield bundle. This approach assures each bundle has approximately equal weight and even coverage of the field, to the extent allowed by the relatively small number of observations. We chose $N_{\text {bundles }}=30$ to balance total number with uniformity across the bundles. 


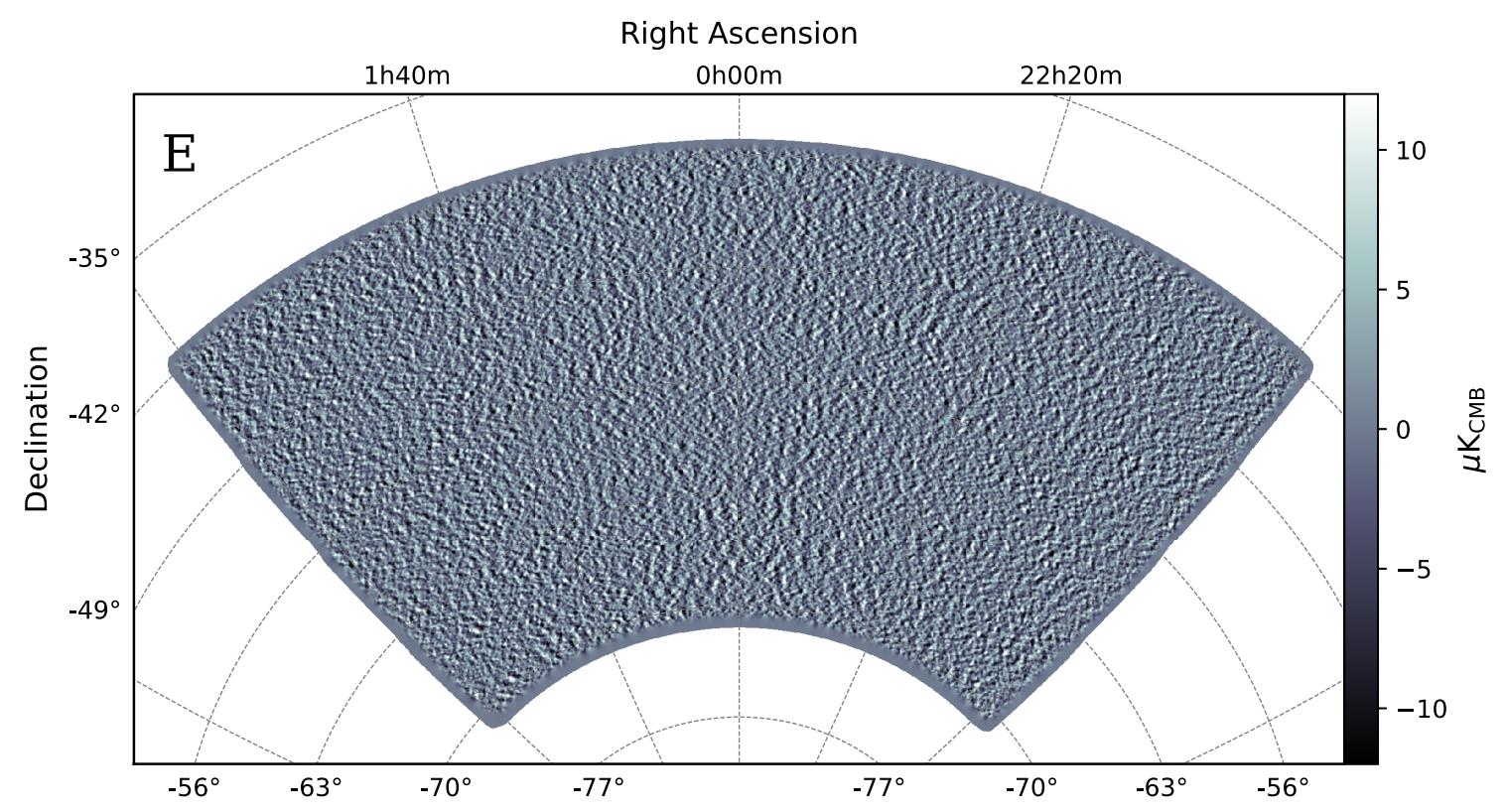

FIG. 3. SPT-3G $2018150 \mathrm{GHz} E$-mode polarization map. The data have been filtered to remove features larger than $\sim 0.5^{\circ}$, and the map has been smoothed by a $6^{\prime}$ FWHM Gaussian.
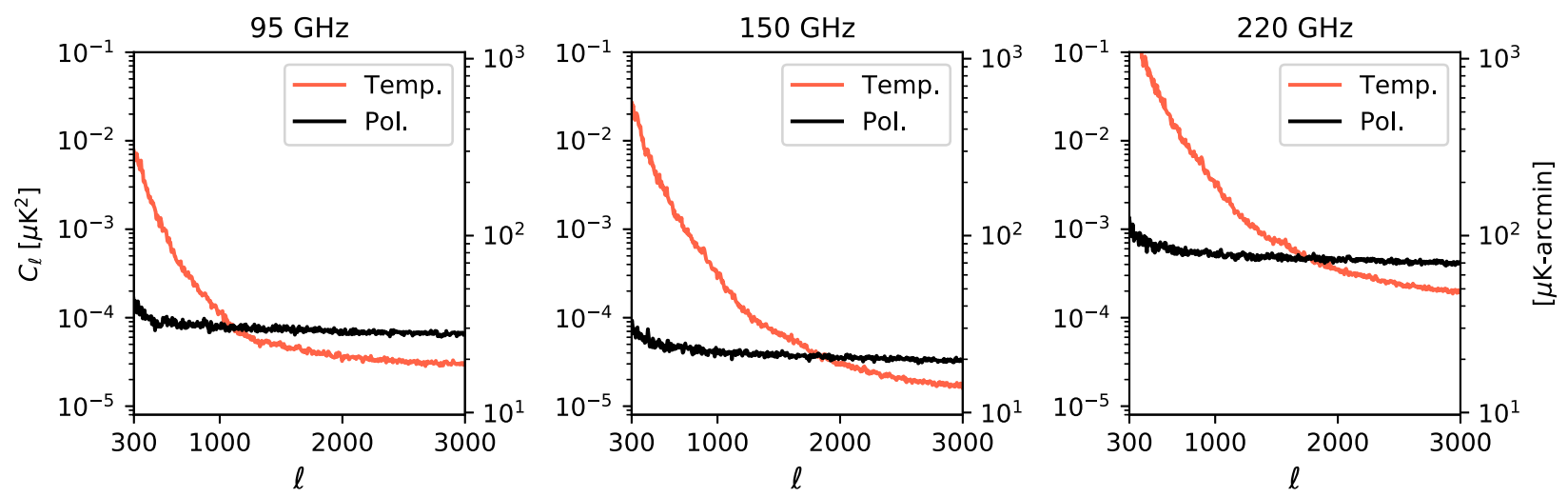

FIG. 4. Temperature and polarization noise power spectra, corrected for the transfer functions of TOD processing. In each subplot, the left-hand vertical axis displays the noise in units of $\mu \mathrm{K}^{2}$, while the right-hand vertical axis displays the equivalent map depth in units of $\mu \mathrm{K}$-arcmin.

\section{POWER SPECTRUM}

We calculate power spectra from the maps in the flat-sky approximation, in which we relate the Fourier wave numbers $\left(k_{x}, k_{y}\right)$ to angular multipole via $|\mathbf{k}|=\ell$. We rotate curved-sky $Q$ and $U$, defined along the longitudes and latitudes on a sphere, to flat-sky $Q^{\prime}$ and $U^{\prime}$, defined along the vertical and horizontal axis of a flat map, by

$$
\begin{aligned}
& Q^{\prime}=Q \cos \left(2 \psi_{\alpha}\right)+U \sin \left(2 \psi_{\alpha}\right), \\
& U^{\prime}=-Q \sin \left(2 \psi_{\alpha}\right)+U \cos \left(2 \psi_{\alpha}\right),
\end{aligned}
$$

where $\psi_{\alpha}$ is the angle measured from the vertical axis to north for pixel $\alpha$ as defined by the map projection. The
Fourier transforms of the rotated $Q^{\prime}$ and $U^{\prime}$ maps are then used to construct the Fourier-space $E$-mode map via [44]

$$
E_{\ell}=Q_{\ell}^{\prime} \cos 2 \phi_{\ell}+U_{\ell}^{\prime} \sin 2 \phi_{\ell},
$$

where $\boldsymbol{\ell}=\left(\ell_{x}, \ell_{y}\right)$ and $\phi_{\ell}=\arctan \left(-\ell_{x} / \ell_{y}\right)$.

\section{A. Cross-spectra}

Following prior SPT analyses, we use the pseudo- $C_{\ell}$ method to compute binned power spectrum estimates, or "band powers," and use a cross-spectrum approach [45,46] to eliminate noise bias. We compute cross-spectra between pairs of bundles by first multiplying each map by an apodization mask $\mathbf{W}$, with the product denoted as $\mathbf{m}_{A}^{X, \nu_{i}}$, 
where $X \in\{T, E\}, A$ indexes bundle number, and $i$ indexes frequency band. We then compute sets of cross-spectra via

$$
\tilde{D}_{b, A \times B}^{X Y, \nu_{i} \times \nu_{j}}=\frac{1}{N_{b}} \sum_{\ell \in b} \frac{\ell(\ell+1)}{2 \pi} \operatorname{Re}\left[m_{\ell, A}^{\left(X, \nu_{i}\right)} m_{\ell, B}^{\left(Y, \nu_{j}\right) *}\right],
$$

for all bundles $A \neq B$, where $N_{b}$ is the number of modes in each $\ell$-bin $b$. The average of all cross-spectra for a given spectrum and frequency combination is then used to obtain the final band powers, $\tilde{D}_{b}^{X Y, \nu_{i} \times \nu_{j}}$. As is customary, here we report power spectra using the flattened spectrum, defined as

$$
D_{\ell} \equiv \frac{\ell(\ell+1)}{2 \pi} C_{\ell}
$$

\section{B. Unbiased spectra}

To obtain unbiased estimates of power spectra, we follow the MASTER algorithm (Ref. [47], hereafter H02), briefly summarized here. The power spectra of maps constructed as described above yield estimates of the true $C_{\ell}$ that have been biased by TOD- and map-level processing. These biased or pseudo- $C_{\ell}$, denoted by $\tilde{C}_{\ell}$, and the true $C_{\ell}$ are related via

$$
\left\langle\tilde{C}_{\ell}\right\rangle=\sum_{\ell^{\prime}} M_{\ell \ell^{\prime}} F_{\ell^{\prime}} B_{\ell^{\prime}}^{2}\left\langle C_{\ell^{\prime}}\right\rangle
$$

in which the brackets denote ensemble averages, $B_{\ell}$ describes the effects of the instrument beam and map pixelization, $F_{\ell}$ is a transfer function encapsulating the effects of TOD filtering, and $M_{\ell \ell^{\prime}}$ is a matrix describing the mixing of power that results from incomplete sky coverage.

Following H02, we introduce the binning operator $P_{b e}$ and its inverse operation $Q_{\ell b}$ : if we write the binned equivalent of Eq. (5) utilizing the shorthand $K_{\ell \ell^{\prime}} \equiv$ $M_{\ell \ell^{\prime}} F_{\ell^{\prime}} B_{\ell^{\prime}}^{2}$ and $K_{b b^{\prime}} \equiv P_{b \ell} K_{\ell \ell^{\prime}} Q_{\ell^{\prime} b^{\prime}}$, then an unbiased estimator of the true power spectrum can be calculated from the pseudospectra via

$$
\widehat{C_{b}}=K_{b b^{\prime}}^{-1} P_{b^{\prime} \ell^{\prime}} \tilde{C}_{\ell} .
$$

To compare the unbinned theory $C_{\ell}^{\text {th }}$ to our band powers, we compute the binned theory spectra as $C_{b}^{\text {th }}=W_{b \ell} C_{\ell}^{\text {th }}$, where $W_{b e}$ are the band-power window functions defined as

$$
W_{b \ell}=K_{b b^{\prime}}^{-1} P_{b^{\prime} \ell^{\prime}} K_{\ell^{\prime} \ell} .
$$

\section{Mask and mode-coupling}

Prior to computing their Fourier transforms, we multiply the maps by an apodization mask $\mathbf{W}$ to smoothly roll off the map edges to zero and remove excess power from bright point sources. The apodization mask is generated in much the same manner as in H18, using the same mask for all map bundles across all frequency bands. First, a binary mask is created for each bundle by smoothing the coadded bundle weights with a $5^{\prime}$ Gaussian, then setting to zero any pixels with a weight below $30 \%$ of the median map weight. The intersection of all the bundle masks is then edgesmoothed with a $30^{\prime}$ cosine taper. Point sources detected above $50 \mathrm{mJy}$ at $150 \mathrm{GHz}$ are masked with a $5^{\prime}$ radius disk (the same size mask used during TOD processing), and the cutouts edge-smoothed with a $10^{\prime}$ cosine taper. The effective area of the final mask, defined as $\sum \mathbf{W}^{2} A_{\alpha}$ where $A_{\alpha}=4 \operatorname{arcmin}^{2}$ is the area of each pixel, is equal to $1614 \mathrm{deg}^{2}$. This area is larger than the stated survey size as a result of the inclusion of lower-weight regions along the map boundaries.

Applying a real-space apodization mask, or imposing any survey boundary, convolves the Fourier transform of the effective mask with that of the on-sky signal, coupling power between formerly independent $\ell$-modes. This effect is encapsulated in the mode-coupling matrix $M_{\ell \ell^{\prime}}$. Previous SPT analyses have used an analytic calculation of the mode-coupling matrix in the flat-sky regime, as derived in H02 for temperature and the Appendix of Ref. [41] (hereafter C15) for polarization (for notational simplicity we omit the $X Y$ superscript on $M_{\ell \ell^{\prime}}$, though separate matrices for $T E$ and $E E$ are used in the analysis). In H18 this calculation was further verified for the input range $0<$ $\ell<500$ with the use of curved-sky HEALPix ${ }^{4}[48,49]$ simulations.

Here we employ an alternate means of simulating $M_{\ell \ell^{\prime}}$ that additionally captures distortions due to the map projection. A set of HEALPix skies are generated in a similar manner as in $\mathrm{H} 18$, with each realization formed from an input spectrum set to zero outside of a selected $\Delta \ell=5$ bin; however, here the curved-sky maps are then reprojected to our flat map projection before applying the apodization mask. The power spectrum is then computed in the usual manner, revealing to which multipoles the $\Delta \ell=5$ input power has been mixed. One full realization of the mode-coupling matrix requires 640 individual simulations to cover the range $0<\ell<3200$ in increments of $\Delta \ell=5$, and 150 such realizations are averaged to obtain the final mode-coupling matrix $M_{\ell \ell^{\prime}}$.

\section{Transfer function}

The filter transfer function $F_{\ell}$ captures the effects of the filtering steps discussed in Sec. IIIC. $F_{\ell}$ is obtained through simulations, discussed further in Sec. IV D 1. In brief, a known input spectrum $C_{\ell}^{\text {th }}$ is used to generate $\mathcal{O}(100$ s) of sky realizations and simulated TOD, to which are then applied the same filtering steps as on the real data.

\footnotetext{
${ }^{4}$ http://healpix.sf.net/.
} 
The output spectra are then compared to the input spectra to obtain the effects of TOD filtering.

Solving Eq. (5) for $F_{\ell}$ directly would necessitate inverting $M_{\ell \ell^{\prime}}$, which may be ill conditioned. Instead, we iteratively solve for $F_{\ell}$ using the method prescribed in H02:

$$
\begin{aligned}
F_{\ell}^{(0)} & =\frac{\left\langle\tilde{C}_{\ell}^{\mathrm{sim}}\right\rangle}{w_{2} B_{\ell}^{2} C_{\ell}^{\mathrm{th}}}, \\
F_{\ell}^{(i+1)} & =F_{\ell}^{(i)}+\frac{\left\langle\tilde{C}_{\ell}^{\mathrm{sim}}\right\rangle-M_{\ell \ell^{\prime}} F_{\ell}^{(i)} B_{\ell}^{2} C_{\ell}^{\mathrm{th}}}{w_{2} B_{\ell}^{2} C_{\ell}^{\mathrm{th}}},
\end{aligned}
$$

where $w_{2} \equiv \frac{1}{\Omega} \int d^{2} r \mathbf{W}^{2}$ and $\Omega$ is the area of the map in steradians. We find three iterations sufficient to achieve a stable result.

The iterative approach is unstable for the $T E$ power spectrum due to zero crossings, so instead we use the geometric mean of the $T T$ and $E E$ transfer functions in the same manner as C15 and H18. For cross-frequency power spectra, a transfer function is computed directly for each $\nu_{i} \times \nu_{j}$ spectrum. The $T E$ and $E E$ transfer functions for $150 \mathrm{GHz}$ are shown in Fig. 5, with similar results found for 95 and $220 \mathrm{GHz}$. The difference between the $T E$ and $E E$ transfer functions primarily arises from the $\mathrm{CM}$ filter, which removes large-scale power from temperature while preserving it in polarization. This also causes $\sim 10 \%$ differences in $F_{\ell}$ between the three frequency bands for $\ell<1000$, which diminishes to $<1 \%$ at higher multipoles.

\section{Simulations}

To create the simulations used for recovering the effect of TOD- and map-level processing on the data, we first generate 250 Gaussian realizations of the CMB described by the best-fit $\Lambda$ CDM model to the base_plikHM_ TTTEEE_lowl_lowE_lensing Planck data set [26]. To these we add foreground contributions using two methods. For foreground components expected to be roughly Gaussian distributed (such as the thermal and kinetic SZ effects), we create Gaussian realizations of power spectra from Ref. [50]. These realizations are correlated between frequencies. We also add Poisson-distributed foregrounds according to source population models from Ref. [51] for radio galaxies and from Ref. [52] for dusty star-forming galaxies, with polarization fractions from Ref. [9] and fluxfrequency scaling relations from Ref. [53]. We neglect Galactic foregrounds for these simulations, as the expected polarized power from dust within our survey region is $1-2$ orders of magnitude smaller than the $E$-mode signal over the multipoles and observing frequencies considered here (Galactic dust is accounted for in the likelihood; see Sec. VI). The $T E$ power for all simulated foregrounds is set to zero. These simulated components are then combined in multipole space and multiplied by a Gaussian approximation of the SPT-3G beam (see Sec. IV E), with FWHMs

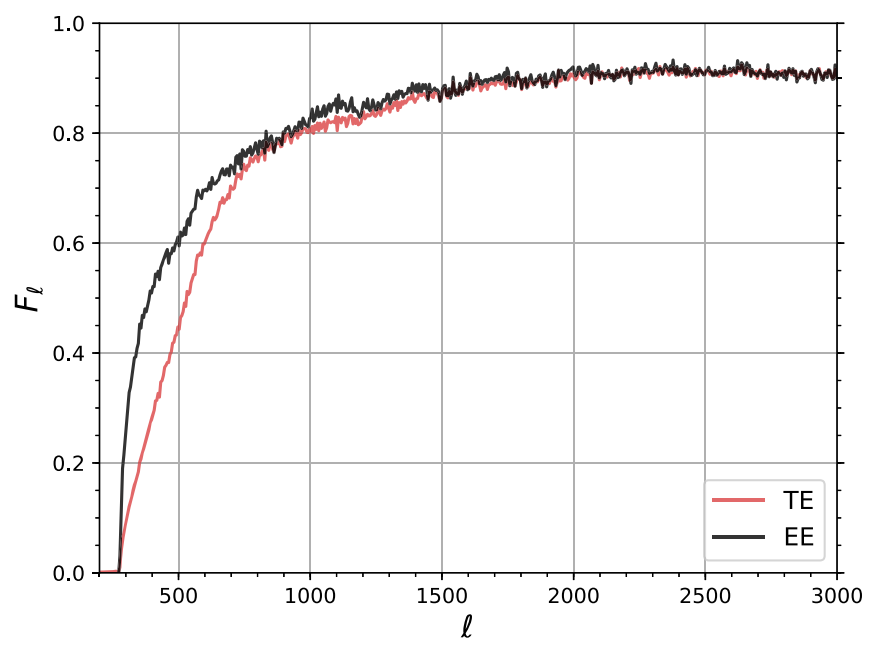

FIG. 5. Filter transfer functions for $150 \mathrm{GHz} T E$ and $E E$ power spectra, computed using 250 TOD simulations of the full SPT-3G 2018 data set. The difference between the $T E$ and $E E$ transfer functions is caused by the common-mode filter.

of $1.7^{\prime}, 1.4^{\prime}, 1.2^{\prime}$ at $95,150,220 \mathrm{GHz}$, respectively, before generating real-space HEALPix sky realizations. These noiseless mock skies are then used along with recorded telescope pointing information from every 2018 subfield observation to generate simulated detector TOD, which are then processed using the same detector cuts and filtering as applied to the real data. The resulting "mock observations" are then bundled and analyzed in exactly the same manner as the real data.

\section{E. Beam}

The beam describes the optical response of the instrument as a function of angle. The maps produced are a convolution of the beam with the underlying sky, equivalently described as a multiplication in Fourier space by the beam window function $B_{\ell} . B_{\ell}$ is estimated in a similar manner to the composite beam analyses in Refs. [40,41,54], using point sources in the $1500 \mathrm{deg}^{2}$ field and five dedicated Mars observations taken during 2018. As in those analyses, we have treated the beam as axially symmetric. The errors induced by this approximation are entirely negligible, as determined using the formalism of Ref. [55] and the known properties of the SPT beam.

The Mars data are convolved with a Gaussian estimate of the telescope pointing jitter (approximately 12" rms) derived from the fitted locations of point sources in individual observations. The brightness of Mars produces a high signal-to-noise beam template out to tens of arcminutes away from the peak response; however, we observe significant evidence for detector nonlinearity at the peak response in the planet scans. To avoid this, the Mars maps are first produced individually for left-going and right-going scans, and any data taken in a scan after Mars passes within $\sim 1$ beam FWHM is masked, as the falling 


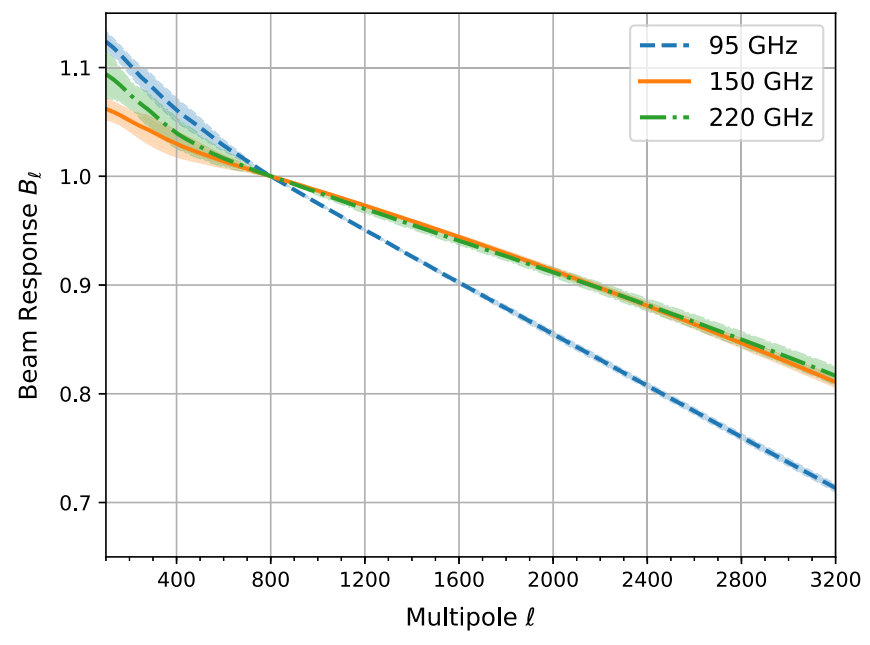

FIG. 6. One-dimensional multipole-space representation of the measured instrument beam, $B_{\ell}$, with uncertainties indicated by the shaded regions. The data are normalized to unity at $\ell=800$.

edge of the beam response is most prone to contamination from detector nonlinearity.

The hole at the location of the peak planet response is filled in by stitching a coadd of point sources that has been convolved with the Mars disk. The stitching operation simultaneously fits a relative scale and offset between the two beam observations using an annular region where both measurements have high signal-to-noise. $B_{\ell}$ is then taken to be the square root of the azimuthal average of the twodimensional (2D) power spectrum of the composite map, after correcting for the planet disk and pixel window functions. The normalization of the beam response is defined by the map calibration procedure described in Sec. IV F 1.

$B_{\ell}$ and uncertainties for the three frequencies are shown in Fig. 6. Over the range of multipoles relevant for this analysis, the fractional beam uncertainty is less than $1.5 \%$. The beam covariance is derived from a set of alternate $B_{\ell}$ curves produced by varying the subfield from which the field sources are drawn, varying which of the five planet observations is used, and sampling from the nominal covariance of the stitching scaling and offset parameters. The beam covariance is then added to the band-power covariance matrix, discussed in Sec. IV H.

\section{F. Absolute calibration}

\section{Subfield calibration}

As this work references separate HII regions for calibrating different halves of the survey field, we calculate and apply a temperature calibration factor for each subfield individually before coadding observations from the four subfields into a single map. To set the individual temperature calibrations, we compute cross-spectra between our subfield temperature maps and the Planck PR 3 maps $^{5}$ of the nearest frequency channel, using 100,143, and $217 \mathrm{GHz}$ for our 95, 150, and $220 \mathrm{GHz}$ bands, respectively.

The Planck maps are mock observed with TOD filtering identical to the real data, though with larger masked regions around point sources to account for the larger Planck beam. An apodization mask with larger point source cutouts is applied to both the mock-Planck and SPT maps, and the corresponding mode-coupling matrix $M_{\ell, \ell^{\prime}}^{\mathrm{ps}}$ is used. We compute the Planck-only and SPT-only power spectra using cross-spectra between half-depth maps from the respective experiments, and we compute the cross-spectra between the two experiments using full-depth maps. We divide out the binned mode-mixing matrix to account for the cut sky and source masking, and compute the binned ratio of the power spectra

$$
\epsilon_{b}=\frac{P_{b, \ell} B_{\ell}^{\mathrm{Planck}}\left(P_{b, \ell} M_{\ell, \ell^{\prime}}^{\mathrm{ps}} Q_{\ell^{\prime}, b^{\prime}}\right)^{-1} \tilde{D}_{b^{\prime}}^{\mathrm{SPT}_{1} \times \mathrm{SPT}_{2}}}{P_{b, \ell} B_{\ell}^{\mathrm{SPT}}\left(P_{b, \ell} M_{\ell, \ell^{\prime}}^{\mathrm{ps}} Q_{\ell^{\prime}, b^{\prime}}\right)^{-1} \tilde{D}_{b^{\prime}}^{\mathrm{SPT} \times \text { Planck }}} .
$$

The average of this ratio over $400 \leq \ell \leq 1500$ is used to set the relative temperature calibration between subfields. All subfield calibration factors are within $\lesssim 7 \%$ of unity, consistent with the expected accuracy of the calibration procedure described in Sec. III B.

We establish uncertainties on the above ratio by combining a single $\Lambda$ CDM sky realization with FFP10 noise simulations for Planck and sign-flip noise realizations for SPT, generated by coadding real SPT-3G data maps with random signs. We compute several similar ratios using other combinations of Planck and SPT data to form the cross-spectra as a data systematics and pipeline consistency check. We find agreement to $\lesssim 1 \%$ in the ratios across different data spectra inputs over the multipole range considered. The beam measured in this manner also serves as a cross-check of our low- $\ell$ beams; while the results are consistent with the position-space measurement, they are less sensitive as a result of the Planck beam size and map noise, and are therefore not used to constrain the shape of the beam response.

\section{Full-field calibration}

We determine the final calibration of the SPT-3G temperature and $E$-mode maps by comparing the measured SPT-3G $T T$ and $E E$ power spectra to the full-sky, foreground-corrected Planck power spectra. Note that while the map calibration described above is expected to be accurate at the percent level, that procedure does not address the absolute amplitude of the $Q$ and $U$ polarization maps. This motivates the $E E$ power spectrum comparison. While not strictly necessary, we also adjust the temperature

\footnotetext{
${ }^{5}$ https://pla.esac.esa.int/.
} 
calibration to be based on the power spectrum comparison for symmetry.

We calculate calibration factors for each frequency band for the temperature (e.g., $T_{\mathrm{cal}}^{95 \mathrm{GHz}}$ ) and $E$-mode (e.g., $\left.E_{\text {cal }}^{95 \mathrm{GHz}}\right)$ maps. The cross-spectra calibration factors are then $T E \propto\left(T_{\text {cal }} E_{\text {cal }}\right)$ and $E E \propto\left(E_{\text {cal }} E_{\text {cal }}\right)$. The calibration factors are constructed based on comparing the Planck combined CMB-only power spectra to the SPT-3G $95 \times 95,150 \times 150$, and $220 \times 220$ band powers over the angular multipole range $300 \leq \ell \leq 1500$ using the Planck bin width of $\Delta \ell=30$. We apply the SPT-3G band-power window functions to the unbinned Planck spectra for this comparison. For temperature, we also account for foreground contamination by subtracting from the SPT-3G band powers the best-fit foreground model from Ref. [3] with additional radio galaxy power from the different point source mask threshold calculated according to the model in Ref. [51]. The foreground corrections are negligible for the $E E$ spectra. We account for the uncertainties on the band-power measurements in this comparison using the covariance described in Sec. IV H as well as the uncertainties on the Planck spectra. We also include the correlated uncertainties in the calibration factors due to the overall Planck absolute calibration uncertainty (taken to be $0.25 \%$ at the map level) and the common sample variance and Planck noise uncertainty across the three frequencies for the $E E$ and $T T$ comparisons.

The adjustments to the $T_{\text {cal }}$ factors recomputed in this manner are all within $\sim 1 \%$ of unity, while the $E_{\text {cal }}$ factors, which may be thought of as the inverse of the effective polarization efficiencies, are 1.028, 1.057, and 1.136 for 95 , 150 , and $220 \mathrm{GHz}$, respectively. That $E_{\text {cal }}$ is a larger correction than $T_{\text {cal }}$ is to be expected, as we do not have per-detector measurements of polarization properties, and instead rely on the as-designed values. We note that despite this, the polarization calibration factors found here are of roughly the same size as those required for SPTpol in C15 and $\mathrm{H} 18$, which did make use of such per-detector polarization information.

The calibration factors are applied to the maps before calculation of the final band powers, and we include all six calibration parameters as nuisance parameters in the likelihood when fitting for cosmology, using priors centered on unity and with widths based on the calculated covariance matrix. The uncertainties on the six calibration parameters are given alongside those of other nuisance parameters in Sec. VI.

\section{G. $T$-to-P leakage}

\section{Monopole deprojection}

Polarization data can be contaminated by leaked temperature signal caused by a variety of factors, including mismatched gain between detectors in a polarization pair and differential beam shapes. As in $\mathrm{C} 15$ and H18, we perform a monopole deprojection, in which a scaled copy of the $T$ map is removed from the $Q$ and $U$ maps. We neglect higher-order leakage terms, as they typically become relevant near the beam scale $(\ell \sim 11000)$, while this analysis extends only to $\ell=3000$.

In both $\mathrm{C} 15$ and $\mathrm{H} 18$, the monopole leakage coefficients $\epsilon^{P}$, where $P \in\{Q, U\}$, were calculated by directly comparing the respective $C_{\ell}^{T P}$ to $C_{\ell}^{T T}$ over some range of $\ell$, and the deprojected maps obtained via $P^{\prime}=P-\epsilon^{P} T$. The same method used in this analysis would be biased by the highpass TOD filter, due to the following effect. In the 2D Fourier plane, $Q Q$ power is oriented along the $\ell_{x}$ and $\ell_{y}$ axes while $U U$ power is oriented at $45^{\circ}$. As the temperature signal is uncorrelated with $Q$ and $U$ across the sky, the azimuthal average of the $T Q$ and $T U$ correlations should be zero (i.e., at each $\ell$, the orthogonal lobes of power in the $2 \mathrm{D}$ Fourier plane are of equal magnitude but opposite sign). However, as the telescope scanning direction is along $\ell_{x}$, the high-pass filter removes power from low- $\ell_{x}$ modes, leaving a residual signal in the $T Q$ azimuthal average that is highly correlated with $T E$. As $T U$ modes are oriented primarily at $45^{\circ}$ in the $2 \mathrm{D}$ Fourier plane, the loss of $\ell_{x}<$ 300 power does not change their net-zero azimuthal average.

To account for the correlation with $T E$, we fit each of $T Q$ and $T U$ to a linear combination of $T E$ and $T T$ according to

$$
C_{\ell}^{T P}=\epsilon^{P, T T} C_{\ell}^{T T}+\epsilon^{P, T E} C_{\ell}^{T E} .
$$

The $\epsilon^{P, T T}$ coefficients are then used for monopole deprojection in the usual fashion, while the $\epsilon^{P, T E}$ values are discarded.

Two tests of this deprojection method are performed before application to data. First we check that the $\epsilon^{P, T T}$ coefficients are consistent with zero in noiseless mock observations. Then, a known amount of $T$-to- $P$ leakage is injected in the simulations to verify it can be recovered. After passing both of these checks, we calculate the leakage coefficients from real data, obtaining the values in Table I. We perform the deprojection on the data, though the resulting shift in band powers is entirely negligible given the reported band-power uncertainties. We accordingly neglect the error on the monopole leakage terms.

\section{Leakage from the common-mode filter}

Another form of $T$-to- $P$ leakage results from the $\mathrm{CM}$ filter. As the polarized power is measured using the difference in signal between orthogonally polarized

TABLE I. $\quad T$-to- $P$ monopole leakage coefficients.

\begin{tabular}{lccc}
\hline \hline & $95 \mathrm{GHz}$ & $150 \mathrm{GHz}$ & $220 \mathrm{GHz}$ \\
\hline$\epsilon^{Q, T T}$ & $0.006 \pm 0.002$ & $0.005 \pm 0.002$ & $0.008 \pm 0.010$ \\
$\epsilon^{U, T T}$ & $0.008 \pm 0.002$ & $0.013 \pm 0.002$ & $0.015 \pm 0.010$ \\
\hline \hline
\end{tabular}


detectors, subtracting the same common mode from all detectors should not affect the measured polarization. However, here we have not enforced explicit pair differencing when making polarized maps, allowing the polarized signal in a given map pixel to be formed from detectors in physically distant focal plane pixels. The CM filter generally removes a different amount of power from two such detectors, thereby affecting the polarization signal. While the CM filter is empirically seen to reduce polarization noise, it also directly injects some fraction of the $\ell \sim 500$ (corresponding to the angular extent of a detector wafer) temperature power into the polarization maps. To quantify this leakage, we mock observe a set of $T$-only simulations and measure the power leaked into $E E$ and $T E$. We find the leakage to depend on the particular configuration of detectors used to form the $\mathrm{CM}$, differing in both sign and magnitude across the three frequency bands, with maximum amplitudes near $\ell=500$ of $0.20 \mu \mathrm{K}^{2}$ for $E E$ and $10 \mu \mathrm{K}^{2}$ for $T E$.

This CM filter-induced $T$-to- $P$ leakage is also present in the simulations used to obtain the filter transfer function. Although $F_{\ell}$ is a multiplicative correction, and this $T$-to- $P$ leakage is an additive bias, to first order $F_{\ell}$ already removes this leakage; when reconstructing the input $D_{\ell, \text { th }}^{E E}$ from simulated $\tilde{D}_{\ell}^{E E}$ using Eq. (5), no residual bias is seen. As will be discussed in Sec. V, realistic changes to the input spectra used for the simulations do not significantly affect $F_{\ell}$, so this bias will already be reduced to a negligible level for $E E$ data.

The leakage in $T E$ is not handled so easily, however, as $F_{\ell}^{T E}$ is not constructed specifically from $T E$ spectra, but rather as the geometric mean of $F_{\ell}^{T T}$ and $F_{\ell}^{E E}$. When reconstructing the input $D_{\ell \text {,h }}^{T E}$ from simulated $\tilde{D}_{\ell}^{T E}$ using Eq. (5), a residual bias remains. The same set of simulations for obtaining $F_{\ell}$ is used to calculate the following residual $T E$ bias, which is then subtracted from the data:

$$
T E_{\mathrm{bias}}=\tilde{D}_{\ell, \mathrm{sim}}^{T E}-\sum_{\ell^{\prime}} M_{\ell \ell^{\prime}} F_{\ell^{\prime}}^{T E} B_{\ell^{\prime}}^{2} D_{\ell, \mathrm{th}}^{T E} .
$$

In addition to the check against varying input simulation spectra discussed below, $T$-only Planck maps corresponding to the SPT-3G coverage region are mock observed to verify the leakage bias in $T E$ to be expected from the real sky, with excellent agreement found between those results and those from the standard set of simulations.

\section{H. Band-power covariance matrix}

The band-power covariance matrix captures the uncertainty in individual band powers and their correlations as well as the correlations between different spectra and different frequency bands. This covariance matrix includes contributions from noise and sample variance. We estimate the noise variance from the set of measured cross-spectra and the sample variance from the set of 250 signal-only simulations. In a final step, the uncertainty from the beam measurement is added. We neglect any contributions from the simulation-derived corrections discussed in the preceding section.

The calculation of the covariance matrix follows the general procedure outlined in the Appendix of Ref. [56]. The three frequency bands are used to form three autofrequency spectra and three cross-frequency spectra for both $E E$ and $T E$, giving the covariance matrix a $12 \times 12$ block structure. The estimate of the covariance is noisy given the finite number of simulations and observations; we therefore "condition" the covariance matrix to reduce noise in both the diagonal and off-diagonal elements.

For the diagonal elements, we expect a fractional uncertainty of $\sqrt{2 / n_{\mathrm{obs}}}$; for the 30 data bundles in this analysis, this is $26 \%$. To mitigate this, we extract the effective number of modes in each $\ell$-bin from the signalonly simulations detailed in Sec. IV D 1, which allows us to compare the poor noise variance estimates to their expectation values. This comparison yields an estimate of the noise spectra, which we smooth with a Gaussian kernel and use to assemble an improved estimate of the noise variance. We add the sample variance contribution to the noise variance to obtain conditioned diagonals for all covariance blocks.

To ameliorate the noise of off-diagonal elements, we condition the underlying correlation matrices. We average the estimated correlation matrices of all 12 on-diagonal blocks and inspect band-diagonal slices (i.e., elements the same distance away from the diagonal). To account for the widening of the mode-coupling kernel over the angular multipole range, we generalize the procedure applied in $\mathrm{C} 15$ and $\mathrm{H} 18$ and fit second-order polynomials to the banddiagonal slices. We replace off-diagonal elements with these fits and set elements further than $\Delta \ell>100$ from the main diagonal to zero as correlations become negligible. The correlation matrix conditioned in this way is then combined with the previously calculated diagonal elements of each block to construct the conditioned covariance matrix.

We have validated this conditioning approach using a suite of 1000 flat-sky, single-frequency simulations that mimic the SPT-3G 2018 data set (30 map bundles, 200 transfer function simulations, $1 / \mathrm{f}$ noise profile matching $\mathrm{H} 18$ with $\mathrm{N}_{\text {white }}^{\mathrm{T}}=10 \mu \mathrm{K}$-arcmin). We measure the $E E$ and $T E$ spectrum for each simulation, estimate the band-power covariance matrix using the distribution of the bundle cross-spectra, and apply the conditioning scheme described above. Comparing the covariance matrices obtained in this way to the average of the unconditioned matrices across all simulations, we find that the residuals along the main diagonals of all covariance blocks are consistent with zero.

We further validate the conditioning scheme by ensuring that its impact on parameter estimation is minimal. We do 
this by considering a $\Lambda \mathrm{CDM}+\mathrm{N}_{\mathrm{eff}}$ model, i.e., introducing the effective number of neutrino species as a free parameter. This is motivated by the signature of changes to $\mathrm{N}_{\text {eff }}$ left in the damping tail of the CMB power spectra, and that by design the devised conditioning scheme smooths noise in the covariance more aggressively at small angular scales. We therefore expect this cosmological model to be a sensitive test of the conditioning step. We find the best-fit $\mathrm{N}_{\text {eff }}$ value while fixing the core $\Lambda \mathrm{CDM}$ parameters to their input values using a Gaussian likelihood for all simulations. We perform this calculation twice for each realization: once using the realization's conditioned band-power covariance matrix and once using the average of all unconditioned covariance matrices. Across the simulations, we find that the standard deviations of the resulting two distributions of best-fit $\mathrm{N}_{\text {eff }}$ values match. Furthermore, the width of the distributions are consistent with a simple Fisher forecast. We observe no evidence that the conditioning procedure introduces a bias to parameter constraints.

The uncertainty from the beam measurement is added to the band-power covariance matrix described above using the same procedure as in Refs. [40,54, C15]. First, we construct a "beam correlation matrix"

$$
\boldsymbol{\rho}_{b b^{\prime}}^{\text {beam }}=\left(\frac{\delta D_{b}}{D_{b}}\right)\left(\frac{\delta D_{b^{\prime}}}{D_{b^{\prime}}}\right)
$$

where

$$
\frac{\delta D_{b}}{D_{b}}=1-\left(1+\frac{\delta B_{b}}{B_{b}}\right)^{-2}
$$

represents the effect of the beam uncertainty $\delta B_{b}$ on the power spectrum. Model band powers $D_{b}$ are then used to generate a covariance from the beam correlation matrix:

$$
\mathbf{C}_{b b^{\prime}}^{\text {beam }}=\boldsymbol{\rho}_{b b^{\prime}}^{\text {beam }} D_{b} D_{b^{\prime}}
$$

Our final results are robust with respect to the beam covariance assumed, with no effect on cosmological constraints after increasing the covariance by a factor of 4 .

\section{TESTS FOR SYSTEMATIC ERRORS}

We perform two primary tests on the data and analysis pipeline, with the first using null tests to probe for systematic effects in the data, and the second verifying the robustness of the pseudospectrum debiasing pipeline against changes to the input power spectrum.

\section{A. Null tests}

To check that the data are free of systematics above the noise level, we perform a series of null tests, in which the data are divided based on a possible source of systematic error, and the groups of data are then differenced to form a collection of null maps. The cross-spectra of the null maps are then compared to the expected null spectrum if that systematic were absent. The expectation spectra are calculated using the same noiseless mock observations detailed in Sec. IV D 1 used for obtaining $F_{\ell}$. The expected null spectra are typically consistent with zero, although differences in e.g., live detector counts can cause nonzero expectation spectra.

We perform the following null tests, most of which have also been explored in prior SPT analyses:

Azimuth: We test for sensitivity to ground signals by ordering the data based on the average azimuth of the observation. We divide azimuth according to the direction of the Dark Sector Laboratory, the building connected to the telescope, which we expect to be the dominant source of any ground-based pickup.

First-Second: This tests for time-dependent effects by ordering the data chronologically into the beginning and end of the season. For 2018, this is degenerate with splitting the data based on if the Sun was below or above the horizon, and therefore tests for both Sun contamination and long time-scale drifts.

Left-Right: This divides each observation into left-going scans and right-going scans, and is intended to test for asymmetric scanning or effects due to the elevation steps.

Moon up-Moon down: We test for additional beam sidelobe pickup by dividing the data based on whether the Moon was above or below the horizon.

Saturation: We test for effects of decreased array responsivity by ordering the data based on the average number of detectors flagged as saturated during an observation.

Wafer: We test for effects due to differing detector properties by dividing the wafers into two groups based on optical response to the calibrator and bolometer saturation power. Separate maps for each observation are made from the two sets of wafers.

With the exception of the Azimuth test, the null tests use the same chronological bundles as used in the crossspectrum calculation. For the Left-Right test, each bundle is separated into left-going and right-going scans, and these are differenced to create the null maps. An analogous procedure is used for the Wafer null test. For the FirstSecond, Moon Up-Moon Down, and Saturation tests, each observation is assigned a value based on the susceptibility of that observation to the potential source of systematic error, and the bundles are then rank ordered by the average of this value across their constituent observations. The halves of the rank-ordered list are then subtracted (i.e., bundle 1 from bundle 16 , bundle 2 from bundle $17, \ldots$, bundle 15 from bundle 30 ) to form the null maps. For the Azimuth test, the normal chronological bundles would average down any potential systematic, as the observing cadence of the telescope effectively randomizes the 
TABLE II. Individual null test PTE values and the combined PTE value for each test across all frequencies and spectra.

\begin{tabular}{|c|c|c|c|c|c|c|c|}
\hline & \multicolumn{2}{|c|}{$95 \mathrm{GHz}$} & \multicolumn{2}{|c|}{$150 \mathrm{GHz}$} & \multicolumn{2}{|c|}{$220 \mathrm{GHz}$} & \multirow{2}{*}{$\frac{\text { Combined Row }}{\text { PTE }}$} \\
\hline & $T E$ & $E E$ & $T E$ & $E E$ & $T E$ & $E E$ & \\
\hline Azimuth & 0.5974 & 0.4939 & 0.1969 & 0.0054 & 0.9023 & 0.8598 & 0.1636 \\
\hline First-Second & 0.3131 & 0.6800 & 0.2594 & 0.9825 & 0.6745 & 0.4779 & 0.7779 \\
\hline Left-Right & 0.3207 & 0.2285 & 0.6895 & 0.6761 & 0.3906 & 0.5617 & 0.6346 \\
\hline Moon Up-Down & 0.8127 & 0.9954 & 0.7333 & 0.4974 & 0.9175 & 0.7619 & 0.9943 \\
\hline Saturation & 0.0962 & 0.8606 & 0.1186 & 0.4727 & 0.6097 & 0.4083 & 0.3320 \\
\hline Wafer & 0.1091 & 0.0038 & 0.4806 & 0.0432 & 0.6597 & 0.5993 & 0.0140 \\
\hline
\end{tabular}

azimuthal range over which the field is observed. The observations are therefore rebundled according to the separation between their mean azimuth and the azimuth corresponding to the Dark Sector Laboratory.

For each null test, we use the average and distribution of all null cross-spectra to compute the chi-square compared to the null expectation spectrum, and we then compute the probability to exceed (PTE) this chi-square value given the degrees of freedom. An exceedingly low PTE or a preponderance of low PTEs indicates the data are in larger disagreement with expectation than random chance would allow. We perform three checks on the collection of PTEs: 1) the entire table of PTE values is consistent with a uniform distribution between 0 and 1 with a KolmogorovSmirnov (KS) test $p$-value $>0.05,2$ ) individual PTE values are larger than $0.05 / N_{\text {tests }}$, and 3) the combination of PTEs in each row using Fisher's method has a PTE above $0.05 / N_{\text {rows }}$. We neglect correlations between PTE values when performing these tests, which has the effect of strengthening the KS and Fisher tests while weakening the multiple-comparisons-corrected individual PTE test. These tests and significance thresholds were agreed upon before looking at the collection of final PTEs to avoid confirmation bias.

The null test PTEs are collected in Table II. The distribution of PTEs is consistent with a uniform distribution with a KS test $p$-value of 0.76 . With 36 tests and six rows, the individual PTE threshold is 0.0014 , and the row threshold is 0.0083; although the Azimuth test for $150 \mathrm{GHz}$ $E E$ and Wafer test for $95 \mathrm{GHz} E E$ are marginal, all of the tests pass the agreed-upon criteria, and we conclude that the listed systematics do not affect the data in a statistically significant way.

\section{B. Sensitivity to cosmological model}

Any corrections to the data based on simulations, such as $F_{\ell}$ or additive bias corrections, should be robust against the chosen input cosmology to the simulations. The simulations in Sec. IV D 1 were constructed to match the true sky as closely as possible, so we can be confident that the resulting simulations will yield valid results; however, we still want to test that the pipeline is stable against small variations to the input power spectra.

We create an additional set of simulations with a contrived cosmology chosen to be $\sim 5 \sigma$ discrepant with the results found in $\mathrm{H} 18$, with parameter values $\Omega_{b} h^{2}=0.02$, $\Omega_{c} h^{2}=0.14, H_{0}=61 \mathrm{~km} \mathrm{~s}^{-1} \mathrm{Mpc}^{-1}, \ln \left(10^{10} A_{s}\right)=3.12$, $n_{s}=0.9$, and $\tau=0.06$. Additionally, the foreground power is doubled in comparison to the standard set of simulations. Fifty noiseless realizations of this cosmology are supplied to the mock-observing pipeline, and the resulting $\tilde{C}_{\ell}$ are debiased using the transfer function and $T E$ bias corrections derived from the standard set of simulations. The input spectra are recovered to well within the uncertainties on the reported data band powers, and we therefore find no measurable bias due to $F_{\ell}$ or the $T E_{\text {bias }}$ correction.

\section{PARAMETER FITTING AND MODELING}

We obtain cosmological parameter constraints using the Markov chain Monte Carlo (MCMC) package CosmoMC [57]. ${ }^{6}$ The theoretical CMB spectra are calculated using CAMB [58], ${ }^{7}$ and are modified to account for the effects of instrumental calibration, aberration due to relative motion with respect to the CMB rest frame [59], and super-sample lensing [60]. We also add terms representing Galactic dust emission and polarized dusty and radio galaxies.

We parametrize the $\Lambda \mathrm{CDM}$ model as follows: the density of cold dark matter $\Omega_{c} h^{2}$; the baryon density $\Omega_{b} h^{2}$; the amplitude of primordial density perturbations, $A_{s}$, the tilt of the power spectrum, $n_{s}$, defined at a pivot scale of $0.05 \mathrm{Mpc}^{-1}$; the optical depth to reionization $\tau$; and CosmoMC's internal proxy for the angular scale of the sound horizon at decoupling, $\theta_{M C}$. For the range of angular multipoles considered here, $\tau$ is degenerate with $A_{\mathrm{s}}$; we therefore use large-scale polarization information from Planck [26] to inform a Gaussian prior of $\tau=0.0543 \pm$ 0.0073 , and we report constraints on the combined amplitude parameter $10^{9} A_{\mathrm{s}} e^{-2 \tau}$ in this work. Widening the prior to

\footnotetext{
${ }^{6}$ https://cosmologist.info/cosmomc/.

${ }^{7}$ https://camb.info/.
} 
$\tau=0.065 \pm 0.015$ based on a recent analysis of Planck and WMAP data by Ref. [61] has no significant effect on cosmological parameter constraints.

We account for aberration in a manner similar to Ref. [2] and $\mathrm{H} 18$ by modifying the theory spectrum as

$$
C_{\ell} \rightarrow C_{\ell}-C_{\ell} \frac{d \ln C_{\ell}}{d \ln \ell} \beta\langle\cos \theta\rangle,
$$

where $\beta=1.23 \times 10^{-3}$ is the velocity of the Local Group with respect to the rest frame of the $\mathrm{CMB}$, and $\langle\cos \theta\rangle=$ -0.39 is the mean angular separation between the CMB dipole and the SPT-3G survey field. For super-sample lensing, we follow the procedure laid out by $\mathrm{C} 15$ and $\mathrm{H} 18$, modifying the $\mathrm{CMB}$ spectrum resulting from a set of parameters $\mathbf{p}$ as

$$
\hat{C}_{\ell}^{X Y}(\mathbf{p} ; \kappa)=C_{\ell}^{X Y}(\mathbf{p})-\frac{\partial \ell^{2} C_{\ell}^{X Y}(\mathbf{p})}{\partial \ln \ell} \frac{\kappa}{\ell^{2}},
$$

where the nuisance parameter $\kappa$ quantifies the mean lensing convergence across the survey field. We apply a Gaussian prior on $\kappa$ centered on zero with standard deviation $\sigma_{\kappa}=4.5 \times 10^{-4}$, with the uncertainty estimated from the survey size [60].

The power from Galactic dust is assumed to follow a modified blackbody spectrum with $T_{\text {dust }}=19.6 \mathrm{~K}$ and $\beta_{\text {dust }}=1.59$ and is modeled according to the relation from Refs. [62,63]:

$$
D_{\ell, \text { dust }}^{X Y}=A_{80}^{X Y}\left(\frac{\ell}{80}\right)^{\alpha_{X Y}+2}
$$

where $A_{80}^{X Y}$ is the amplitude of the spectrum at $\ell=80$ at $150 \mathrm{GHz}$, and $\alpha_{X Y}$ is the angular power dust spectral index. Based on Ref. [62], we apply a Gaussian prior on $\alpha_{X Y}$ with a central value of -2.42 and uncertainty 0.02 . We estimate the properties of polarized Galactic dust on the SPT-3G $1500 \mathrm{deg}^{2}$ field using Planck observations in the frequency bands 100, 143, 217, and $353 \mathrm{GHz}$. We assume the aforementioned spectral energy distribution and fit to the amplitude using the ten cross-frequency spectra obtained from an optimal combination of all possible half-mission map cross-spectra. Taking into account Planck color corrections [63], pessimistic calibration errors and assuming the Planck best-fit cosmology, we constrain the amplitude of polarized Galactic dust to be $A_{80}^{E E}=0.095 \pm$ 0.012 and $A_{80}^{T E}=0.184 \pm 0.072$, which we adopt as Gaussian priors in our MCMC analysis. We further check that the constraints remain stable when also fitting for $\beta_{\text {dust }}$ and $\alpha_{E E}$, the fit values of which are in good agreement with our chosen values.

The $E E$ power spectrum of the emission from a Poisson distribution of partially polarized synchrotron and dusty galaxies can be described as
TABLE III. Gaussian priors used for the MCMC fit, including the optical depth to reionization $\tau$, mean-field lensing convergence $\kappa$, the amplitude $A_{80}^{X Y}$ (in $\mu \mathrm{K}^{2}$ ) at $150 \mathrm{GHz}$ and spectral index $\alpha_{80}^{X Y}$ of polarized Galactic dust, the $E E$ power of Poissondistributed point sources $D_{3000}^{\mathrm{ps}, \nu_{i} \times \nu_{j}}$ (in $\mu \mathrm{K}^{2}$ ), absolute temperature calibration factor $T_{\text {cal }}^{\nu_{i}}$, and absolute polarization calibration factor $E_{\mathrm{cal}}^{\nu_{i}}$.

\begin{tabular}{lc}
\hline \hline Parameter & Prior \\
\hline$\tau$ & $0.0543 \pm 0.0073$ \\
$100 \kappa$ & $0 \pm 0.045$ \\
$A_{80}^{E E}$ & $0.095 \pm 0.012$ \\
$\alpha_{E E}$ & $-2.42 \pm 0.02$ \\
$A_{80}^{T E}$ & $0.184 \pm 0.072$ \\
$\alpha_{T E}$ & $-2.42 \pm 0.02$ \\
$D_{3000}^{\mathrm{ps}, 95 \times 95}$ & $0.041 \pm 0.012$ \\
$D_{3000}^{\mathrm{ps}, 150 \times 150}$ & $0.0115 \pm 0.0034$ \\
$D_{3000}^{\mathrm{ps}, 220 \times 220}$ & $0.048 \pm 0.014$ \\
$D_{3000}^{\mathrm{ps}, 95 \times 150}$ & $0.0180 \pm 0.0054$ \\
$D_{3000}^{\mathrm{ps}, 95 \times 220}$ & $0.0157 \pm 0.0047$ \\
$D_{3000}^{\mathrm{ps}, 150 \times 220}$ & $0.0190 \pm 0.0057$ \\
$T_{\mathrm{cal}}^{95} \mathrm{GHz}$ & $1.0 \pm 0.0049$ \\
$T_{\mathrm{cal}}^{150} \mathrm{GHz}$ & $1.0 \pm 0.0050$ \\
$T_{\mathrm{cal}}^{220} \mathrm{GHz}$ & $1.0 \pm 0.0067$ \\
$E_{\mathrm{cal}}^{95} \mathrm{GHz}$ & $1.0 \pm 0.0087$ \\
$E_{\mathrm{cal}}^{150} \mathrm{GHz}$ & $1.0 \pm 0.0081$ \\
$E_{\mathrm{cal}}^{220} \mathrm{GHz}$ & $1.0 \pm 0.016$ \\
\hline \hline
\end{tabular}

$$
D_{\ell}=D_{3000}^{\mathrm{ps}}\left(\frac{\ell}{3000}\right)^{2}
$$

The $T E$ signal from these galaxies is expected to be zero, as the polarization angles are uncorrelated between galaxies. In the baseline case, we apply Gaussian priors to the six $D_{3000}^{\mathrm{ps}, \nu_{i} \times \nu_{j}}$ parameters based on the temperature values from Ref. [3], which we adjust for our flux cut following the model of Ref. [51] and scale by the polarization fractions reported by Ref. [9]. The prior width is dominated by uncertainty in the mean squared polarization fraction, which we conservatively double to yield $30 \%$.

We find that our cosmological parameter constraints are insensitive to the details of the foreground priors, with no significant shifts in the results when the Poisson terms or the polarized Galactic dust amplitudes are doubled or set to zero. We conclude that over our multipole range the band powers are largely insensitive to both of these foreground sources. The priors discussed in this section are summarized in Table III.

We verify that our likelihood is unbiased by analyzing a set of 100 simulated spectra. Mock band powers are created by adding random noise realizations based on our data covariance matrix to the latest Planck best-fit model. We use the likelihood to obtain the best-fit model for each realization, and we find that for all cosmological 
parameters, the mean of the ensemble of simulations lies within one standard error of the input value.

\section{THE SPT-3G 2018 POWER SPECTRA}

\section{A. Band powers}

We present band powers and uncertainties for the six $E E$ and $T E$ cross-frequency power spectra, plotted in Fig. 7 and listed in full in the Appendix. The band powers span the multipole range $300 \leq \ell<3000$, with bin widths of $\Delta \ell=$ 50 for $\ell<2000$ and $\Delta \ell=100$ for $\ell>2000$. The 44 band powers for each spectrum are measured with each of the six frequency combinations of 95, 150, and $220 \mathrm{GHz}$ data, resulting in 528 band-power values in total.

With $150 \times 150 \mathrm{GHz}$ alone, we measure the first seven acoustic peaks of the $E E$ spectrum with 3-4 band powers per peak and signal-to-noise $\geq 6.4$ on each band power. The band powers are sample variance dominated at $\ell<1275$ for $E E$ and $\ell<1425$ for $T E$.

We also construct a set of minimum-variance band powers. Following Ref. [64], the minimum-variance band powers $D^{\mathrm{MV}}$ can be expressed as

$$
D^{\mathrm{MV}}=\left(\mathbf{X}^{\top} \mathbf{C}^{-1} \mathbf{X}\right)^{-\mathbf{1}} \mathbf{X}^{\top} \mathbf{C}^{-1} D .
$$

Here, $D$ and $\mathbf{C}$ are the multifrequency band powers and covariance matrix, and $\mathbf{X}$ is a $528 \times 88$ design matrix, in which each column is equal to 1 in the six elements corresponding to a power spectrum measurement in that $\ell$ space bin and zero elsewhere. In this construction, we have made the simplifying assumption that the polarized foreground power is negligible within the band-power uncertainties. Relative to the most-sensitive single-frequency band, the $150 \times 150 \mathrm{GHz}$ band powers, the minimumvariance band powers have uncertainties 5-10\% smaller at $\ell<1000$ and $20-30 \%$ smaller at $\ell>2000$.

The minimum-variance $E E$ and $T E$ band powers and associated errors are summarized in Table IV and plotted in Fig. 8 along with measurements from several recent experiments. These minimum-variance band powers, measured using only four months of SPT-3G data with slightly over half the number of detectors relative to subsequent observing seasons, are already the most constraining measurements made to date by an instrument on SPT over the multipole ranges $300 \leq \ell \leq 1400$ for $E E$ and $300 \leq$ $\ell \leq 1700$ for $T E$, and are competitive with other current leading measurements.

\section{B. Internal consistency}

The minimum-variance construction above assumes the multifrequency band powers are measuring the same underlying signal and that polarized foregrounds are negligible. We test this assumption by examining the chi-square of the multifrequency band powers to the minimum-variance band powers,

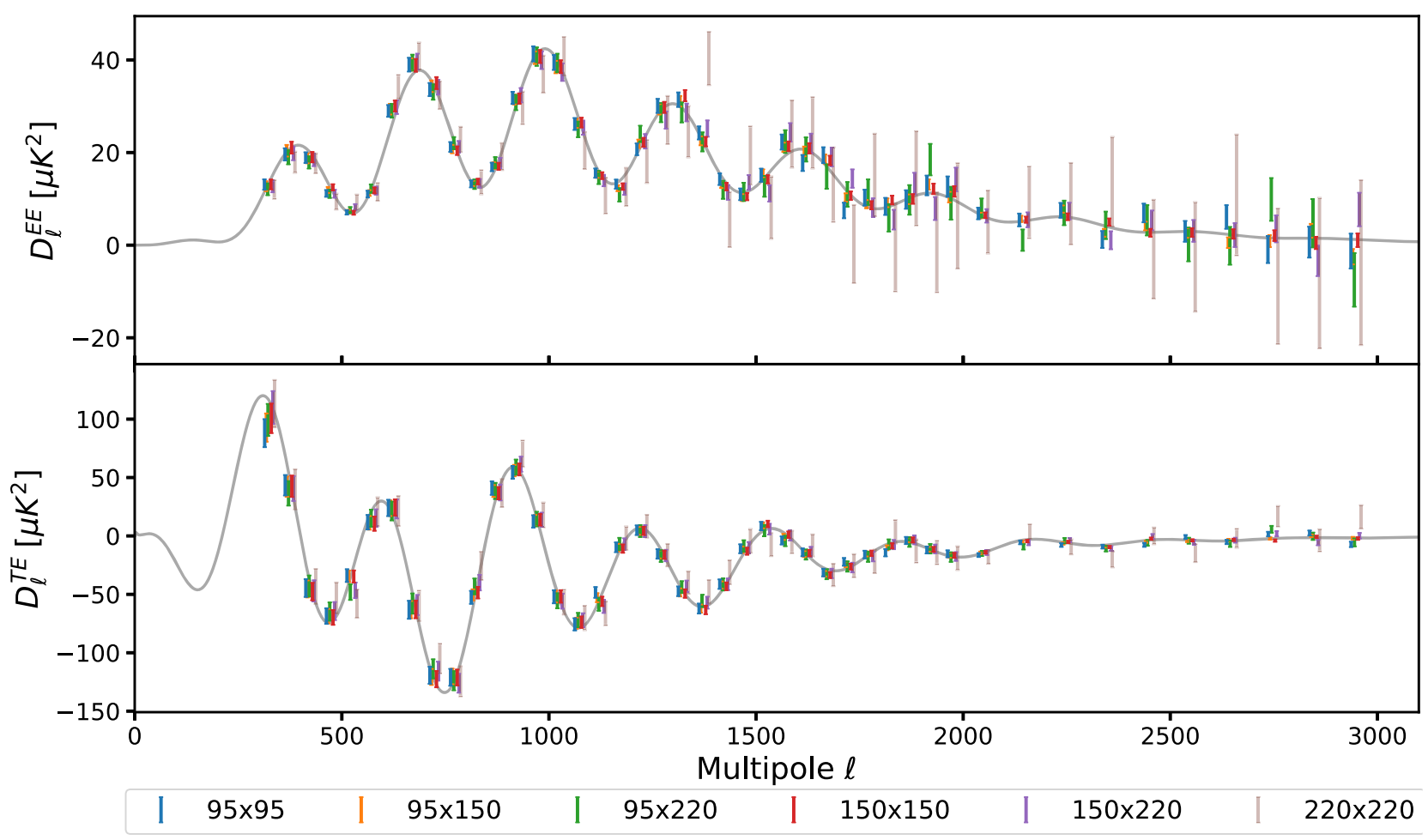

FIG. 7. SPT-3G EE and TE band-power measurements from the six auto- and cross-frequency power spectra overlaid on the Planck best-fit $\Lambda \mathrm{CDM}$ model. The plotted uncertainties are the square root of the diagonal elements of the covariance matrix and do not include beam or calibration uncertainties. A small $\ell$ offset has been applied to each point for plotting purposes. 
TABLE IV. Minimum-variance band powers $D_{b}$ and their associated uncertainties $\sigma$ for the $T E$ and $E E$ power spectra. We also report the band-power window-function-weighted multipole $\ell_{\text {eff }}$ for each $\ell$ range. The band powers and errors are quoted in units of $\mu \mathrm{K}^{2}$. The reported uncertainties are the square root of the diagonal elements of the covariance matrix and do not include beam or calibration uncertainties.

\begin{tabular}{|c|c|c|c|c|c|c|}
\hline$\ell$ Range & $\ell_{\mathrm{eff}}^{T E}$ & $D_{b}^{T E}$ & $\sigma^{T E}$ & $\ell_{\mathrm{eff}}^{E E}$ & $D_{b}^{E E}$ & $\sigma^{E E}$ \\
\hline $300-349$ & 326 & 103.7 & 11.3 & 325 & 14.1 & 1.0 \\
\hline 350-399 & 376 & 39.8 & 8.4 & 375 & 20.4 & 1.2 \\
\hline $400-449$ & 426 & -47.8 & 7.0 & 425 & 19.0 & 1.1 \\
\hline $450-499$ & 475 & -72.1 & 6.0 & 475 & 12.0 & 0.6 \\
\hline $500-549$ & 523 & -35.1 & 4.7 & 524 & 7.2 & 0.4 \\
\hline $550-599$ & 574 & 10.2 & 5.6 & 575 & 11.6 & 0.6 \\
\hline $600-649$ & 625 & 23.6 & 6.6 & 624 & 29.7 & 1.1 \\
\hline 650-699 & 675 & -63.7 & 7.3 & 674 & 39.0 & 1.3 \\
\hline 700-749 & 725 & -120.8 & 6.8 & 725 & 34.5 & 1.2 \\
\hline 750-799 & 774 & -121.2 & 6.6 & 774 & 20.7 & 0.9 \\
\hline $800-849$ & 824 & -49.2 & 4.7 & 824 & 13.5 & 0.6 \\
\hline 850-899 & 874 & 38.0 & 5.0 & 874 & 17.1 & 0.7 \\
\hline 900-949 & 924 & 56.6 & 4.9 & 924 & 31.6 & 1.0 \\
\hline 950-999 & 974 & 13.3 & 4.8 & 974 & 40.6 & 1.3 \\
\hline 1000-1049 & 1024 & -52.3 & 5.2 & 1024 & 38.5 & 1.3 \\
\hline 1050-1099 & 1075 & -74.0 & 4.7 & 1075 & 26.2 & 1.0 \\
\hline 1100-1149 & 1124 & -54.2 & 3.8 & 1124 & 15.0 & 0.6 \\
\hline 1150-1199 & 1174 & -10.0 & 3.3 & 1174 & 12.4 & 0.6 \\
\hline 1200-1249 & 1224 & 4.4 & 3.3 & 1224 & 21.9 & 0.9 \\
\hline 1250-1299 & 1274 & -15.9 & 3.3 & 1275 & 29.2 & 1.1 \\
\hline 1300-1349 & 1324 & -47.8 & 3. & 1325 & 31.1 & 1.1 \\
\hline 1350-1399 & 1374 & -61.7 & 3.4 & 1374 & 22.7 & 0.9 \\
\hline 1400-1449 & 1424 & -42.0 & 3.0 & 1424 & 12.8 & 0.7 \\
\hline 1450-1499 & 1474 & -11.9 & 2.7 & 1474 & 10.6 & 0.6 \\
\hline 1500-1549 & 1524 & & & & & 0.7 \\
\hline 1550-1599 & 1574 & -0.4 & 2.5 & 1574 & 21.4 & 0.9 \\
\hline 1600-1649 & 1624 & -14.7 & 2.4 & 1624 & 20.2 & 0.9 \\
\hline 1650-1699 & 1674 & -32.4 & 2.2 & 1674 & 18.2 & 0.8 \\
\hline 1700-1749 & 1724 & -24.9 & 2.2 & 1724 & 10.3 & 0.7 \\
\hline 1750-1799 & 1775 & -15.2 & 2.0 & 1775 & 8.8 & 0.7 \\
\hline 1800-1849 & 1824 & -9.4 & 1.9 & 1825 & 8.9 & 0.7 \\
\hline 1850-1899 & 1874 & -3.5 & 1.9 & 1874 & 10.0 & 0.8 \\
\hline 1900-1949 & 1924 & -11.3 & 1.8 & 1924 & 12.3 & 0.8 \\
\hline 1950-1999 & 1975 & -16.3 & 1.8 & 1975 & 11.1 & 0.8 \\
\hline 2000-2099 & 2050 & -14.2 & 0.9 & 2049 & 6.4 & 0.4 \\
\hline 2100-2199 & 2151 & -4.8 & 0.9 & 2148 & 5.3 & 0.5 \\
\hline 2200-2299 & 2250 & -5.6 & 0.8 & 2248 & 6.8 & 0.5 \\
\hline 2300-2399 & 2349 & -9.2 & 0.8 & 2348 & 3.5 & 0.5 \\
\hline 2400-2499 & 2450 & -3.6 & 0.8 & 2448 & 3.7 & 0.6 \\
\hline 2500-2599 & 2549 & -3.7 & 0.8 & 2548 & 2.6 & 0.6 \\
\hline 2600-2699 & 2649 & -3.5 & 0.8 & 2648 & 1.9 & 0.7 \\
\hline 2700-2799 & 2749 & -2.1 & 0.8 & 2748 & 1.7 & 0.8 \\
\hline 2800-2899 & 2849 & -0.5 & 0.8 & 2848 & 1.2 & 0.9 \\
\hline 2900-2999 & 2949 & -2.3 & 0.8 & 2948 & -0.1 & 1.0 \\
\hline
\end{tabular}

$$
\chi^{2}=(D-M)^{\mathrm{T}} \mathbf{C}^{-1}(D-M)
$$

where $M=\mathbf{X} D^{\mathrm{MV}}$. We find a $\chi^{2}$ of 438.1 for 440 degrees of freedom (528 multifrequency band powers -88 minimum-
TABLE V. Parameter-level $\chi^{2}$ difference and PTE between subsets of the data and the full data set. We do the comparison in the five-dimensional parameter space, $\left(\Omega_{b} h^{2}, \Omega_{c} h^{2}, \theta_{M C}, 10^{9} A_{s} e^{-2 \tau}, n_{s}\right)$, due to the common $\tau$ prior.

\begin{tabular}{lcc}
\hline \hline Subset & $\chi^{2}$ & PTE \\
\hline$E E$ & 4.69 & $45.45 \%$ \\
$T E$ & 8.96 & $11.06 \%$ \\
$\ell \leq 1000$ & 7.82 & $16.64 \%$ \\
$\ell>1000$ & 7.70 & $17.34 \%$ \\
$95 \mathrm{GHz}$ & 6.68 & $24.57 \%$ \\
$150 \mathrm{GHz}$ & 3.75 & $58.54 \%$ \\
$220 \mathrm{GHz}$ & 2.35 & $79.92 \%$ \\
\hline \hline
\end{tabular}

variance band powers). The PTE for this $\chi^{2}$ is 0.52 . If the $E E$ and $T E$ band powers are evaluated separately, the PTEs are 0.18 and 0.71 , respectively. This indicates that the measurements from different frequency bands and their crosscorrelations are consistent with a common signal, with no evidence for significant contamination due to foregrounds or unmodeled systematics.

We further investigate the internal consistency of the SPT-3G $2018 E E / T E$ data set by subdividing it and examining the parameter constraints from each of the seven data splits: the 95,150 , and $220 \mathrm{GHz}$ auto-frequency spectra, the $\ell<1000$ and $\ell>1000$ data, and the $E E$ and $T E$ spectra individually. We quantify the consistency of each subset with respect to the full model by calculating the parameter-level $\chi^{2}$ and associated PTEs in Table V, following the methodology of Ref. [24]:

$$
\chi^{2}=\Delta \mathbf{p}^{\top} \mathbf{C}_{\mathrm{p}}^{-1} \mathbf{\Delta} \mathbf{p},
$$

where $\Delta \mathbf{p}$ is the vector of parameter differences between the full data set and a given subset. Following Ref. [65], $\mathbf{C}_{\mathrm{p}}$ is the difference of the associated parameter covariance matrices, whereby we account for the correlation between the full data set and the subset. The comparison is carried out over the parameters $\left(\Omega_{b} h^{2}, \Omega_{c} h^{2}, \theta_{M C}, 10^{9} A_{s} e^{-2 \tau}, n_{s}\right)$.

All seven data splits are firmly within the central $95 \%$ confidence interval $[2.5 \%, 97.5 \%]$ and we conclude that there is no evidence for significant internal tension in the data set. We will return to these data splits in Sec. VIII A, when we look at the effect of each subset on the cosmological constraints of the ensemble.

\section{COSMOLOGICAL CONSTRAINTS}

\section{A. SPT-3G}

The cosmological parameter constraints from the 2018 SPT-3G $E E$ and $T E$ multifrequency band powers are summarized in Table VI. We present the one-dimensional (1D) and 2D marginalized posterior probabilities for $\Lambda \mathrm{CDM}$ parameters and $H_{0}$ in Fig. 9. Constraints on nuisance parameters are driven by the priors discussed in 


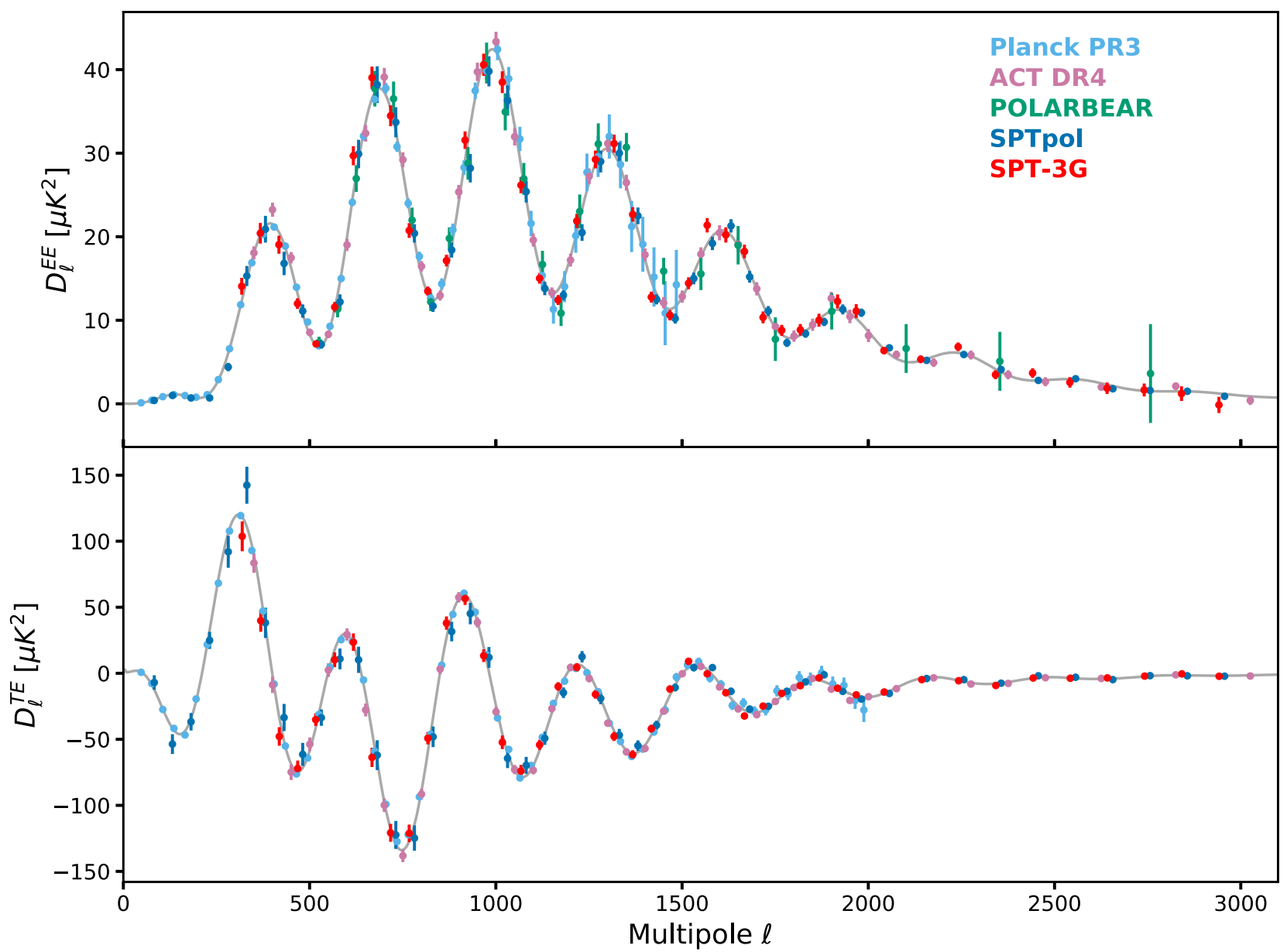

FIG. 8. The minimum-variance SPT-3G $E E$ and $T E$ band powers (red) overlaid on the Planck best-fit $\Lambda$ CDM model, along with the recent measurements from Planck [1], ACT [12], POLARBEAR [15], and SPTpol H18. The Planck EE band powers are restricted to $\ell<1500$. The uncertainties shown for the SPT-3G band powers are the square root of the diagonal elements of the covariance matrix and do not include beam or calibration uncertainties.

TABLE VI. Marginalized constraints and 68\% errors of $\Lambda \mathrm{CDM}$ free and derived parameters from SPT-3G with and without the addition of BAO measurements, from SPT-3G + Planck, and from Planck alone [26]. Best-fit values are given in parentheses. Note that SPT-3G alone does not constrain the optical depth to reionization $\tau$, but uses a Planck-based Gaussian prior of $0.0543 \pm 0.0073$.

\begin{tabular}{|c|c|c|c|c|}
\hline & SPT-3G & $\mathrm{SPT}-3 \mathrm{G} \pm \mathrm{BAO}$ & SPT-3G \pm Planck & Planck \\
\hline \multicolumn{5}{|c|}{ Free } \\
\hline$\Omega_{b} h^{2}$ & $0.02242 \pm 0.00033(0.02243)$ & $0.02240 \pm 0.00032(0.02241)$ & $0.02241 \pm 0.00013(0.0224)$ & $0.02236 \pm 0.00015$ \\
\hline$\Omega_{c} h^{2}$ & $0.1150 \pm 0.0037(0.115)$ & $0.1162 \pm 0.0015(0.1162)$ & $0.1196 \pm 0.0013(0.1195)$ & $0.1202 \pm 0.0014$ \\
\hline $100 \theta_{\mathrm{MC}}$ & $1.03961 \pm 0.00071(1.03964)$ & $1.03951 \pm 0.00066(1.03952)$ & $1.04074 \pm 0.00028(1.04073)$ & $1.04090 \pm 0.00031$ \\
\hline $10^{9} A_{s} e^{-2 \tau}$ & $1.819 \pm 0.038(1.821)$ & $1.826 \pm 0.036(1.826)$ & $1.879 \pm 0.011(1.877)$ & $1.884 \pm 0.012$ \\
\hline$n_{s}$ & $0.999 \pm 0.019(0.999)$ & $0.996 \pm 0.018(0.996)$ & $0.9666 \pm 0.0042(0.9672)$ & $0.9649 \pm 0.0044$ \\
\hline \multicolumn{5}{|l|}{ Derived } \\
\hline$\Omega_{\Lambda}$ & $0.708 \pm 0.020(0.708)$ & $0.7011 \pm 0.0083(0.7014)$ & $0.6867 \pm 0.0077(0.6871)$ & $0.6834 \pm 0.0084$ \\
\hline$H_{0}$ & $68.8 \pm 1.5(68.8)$ & $68.27 \pm 0.63(68.29)$ & $67.48 \pm 0.55(67.49)$ & $67.27 \pm 0.60$ \\
\hline$\sigma_{8}$ & $0.789 \pm 0.016(0.789)$ & $0.7935 \pm 0.0099(0.7933)$ & $0.8084 \pm 0.0069(0.8095)$ & $0.8120 \pm 0.0073$ \\
\hline$S_{8}$ & $0.779 \pm 0.041(0.779)$ & $0.792 \pm 0.018(0.791)$ & $0.826 \pm 0.015(0.827)$ & $0.834 \pm 0.016$ \\
\hline Age/Gyr & $13.808 \pm 0.051(13.807)$ & $13.819 \pm 0.038(13.818)$ & $13.797 \pm 0.022(13.798)$ & $13.800 \pm 0.024$ \\
\hline
\end{tabular}




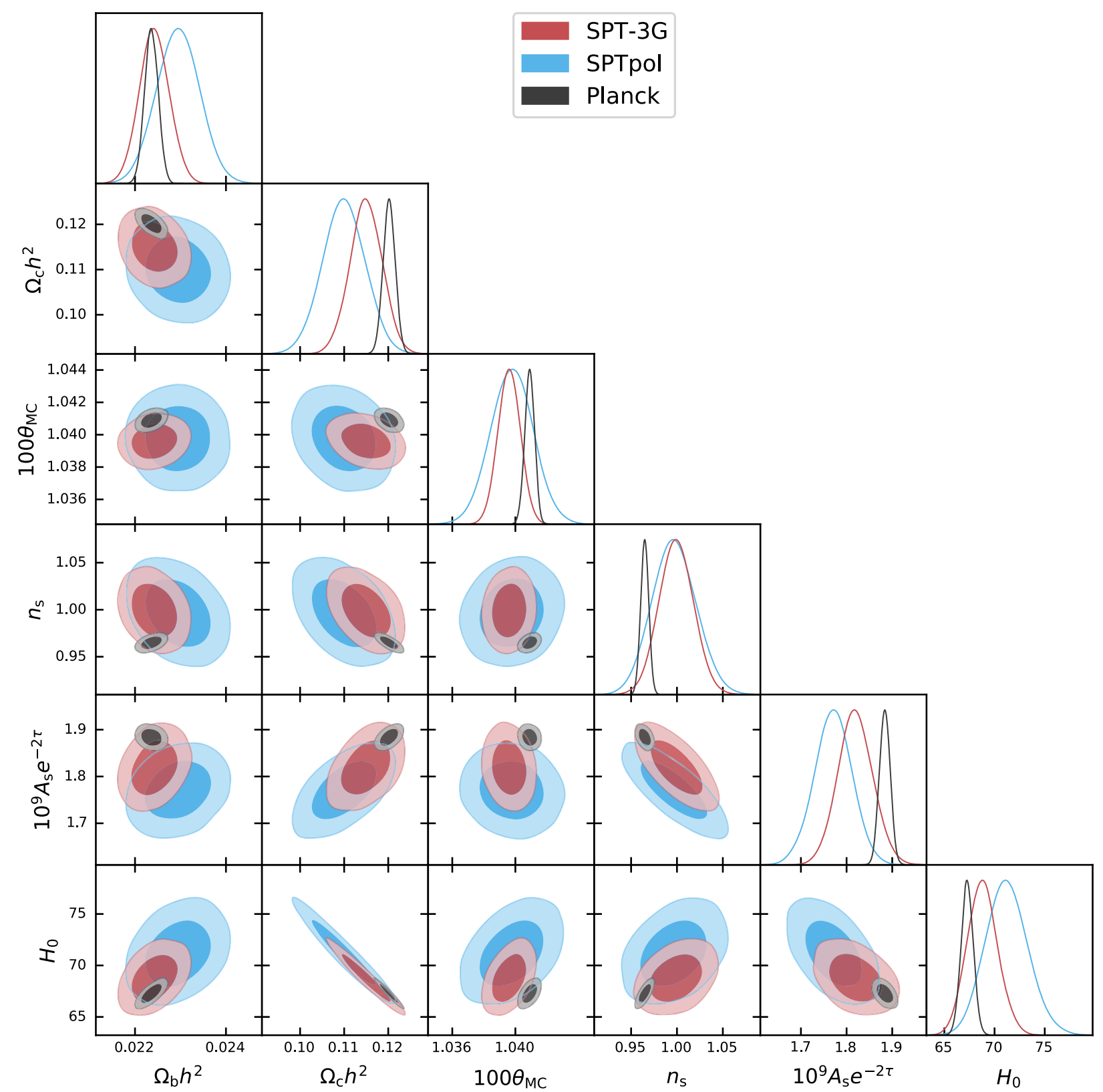

FIG. 9. Marginalized constraints on $\Lambda$ CDM parameters and the Hubble constant for the SPT-3G 2018 EE/TE, SPTpol H18, and Planck [1] data sets. SPT-3G produces consistently tighter constraints than SPTpol. We expect the results of the two analyses to be mildly correlated due to their shared sky area. The results from SPT-3G are statistically consistent with the findings of Planck.

Sec. VI, with all central values well within $1 \sigma$ of their respective prior.

We find the value of the Hubble parameter at present day to be

$$
H_{0}=68.8 \pm 1.5 \mathrm{~km} / \mathrm{s} / \mathrm{Mpc},
$$

in good agreement with other CMB and $\Lambda$ CDM-based measurements $[12,26]$ as well as with local distance ladder measurements calibrated using the tip of the red giant branch (TRGB) [66]. Conversely, this value disagrees at $2.5 \sigma$ with the value of $H_{0}=74.03 \pm 1.42 \mathrm{~km} / \mathrm{s} / \mathrm{Mpc}$ found by Ref. [27] using Cepheid-calibrated distance ladder measurements. It is also $1.8 \sigma$ and $0.9 \sigma$ lower than the value of the Hubble constant measured via the time delays of gravitationally lensed quasars by Refs. [67,68], respectively. Our result represents yet another CMB-based measurement, largely independent of Planck and also relying on $\mathrm{CMB}$ polarization information, that prefers a low value of $H_{0}$ relative to local measurements. 


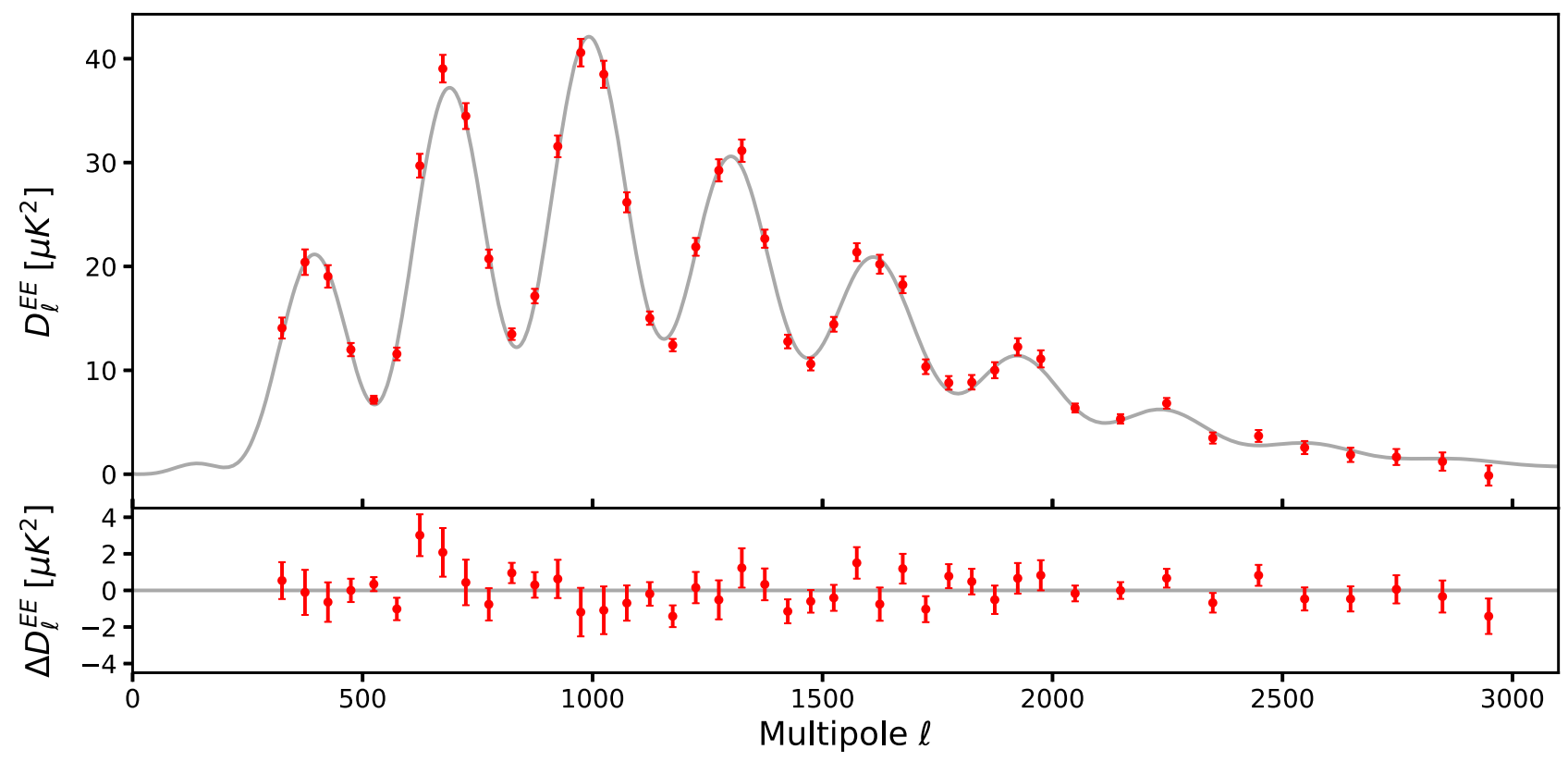

FIG. 10. Minimum-variance $E E$ band powers formed from the six auto- and cross-frequency power spectra and the residuals against the SPT-3G best-fit $\Lambda$ CDM model. Uncertainties are the square root of the diagonal elements of the covariance matrix and do not include beam or calibration uncertainties.

We find the root-mean-square fluctuation in the linear matter density field on $8 \mathrm{Mpc} / h$ scales at present day, $\sigma_{8}$, to be

$$
\sigma_{8}=0.789 \pm 0.016
$$

This is $1.3 \sigma$ lower than the most recent Planck result and $0.3 \sigma$ higher than the joint constraint from the latest SPTpol lensing power spectrum and baryon acoustic oscillation (BAO) data [69], though we expect a mild correlation with the latter result due to the partially shared sky area of the surveys. The SPT3G 2018 value is in good agreement with local structure

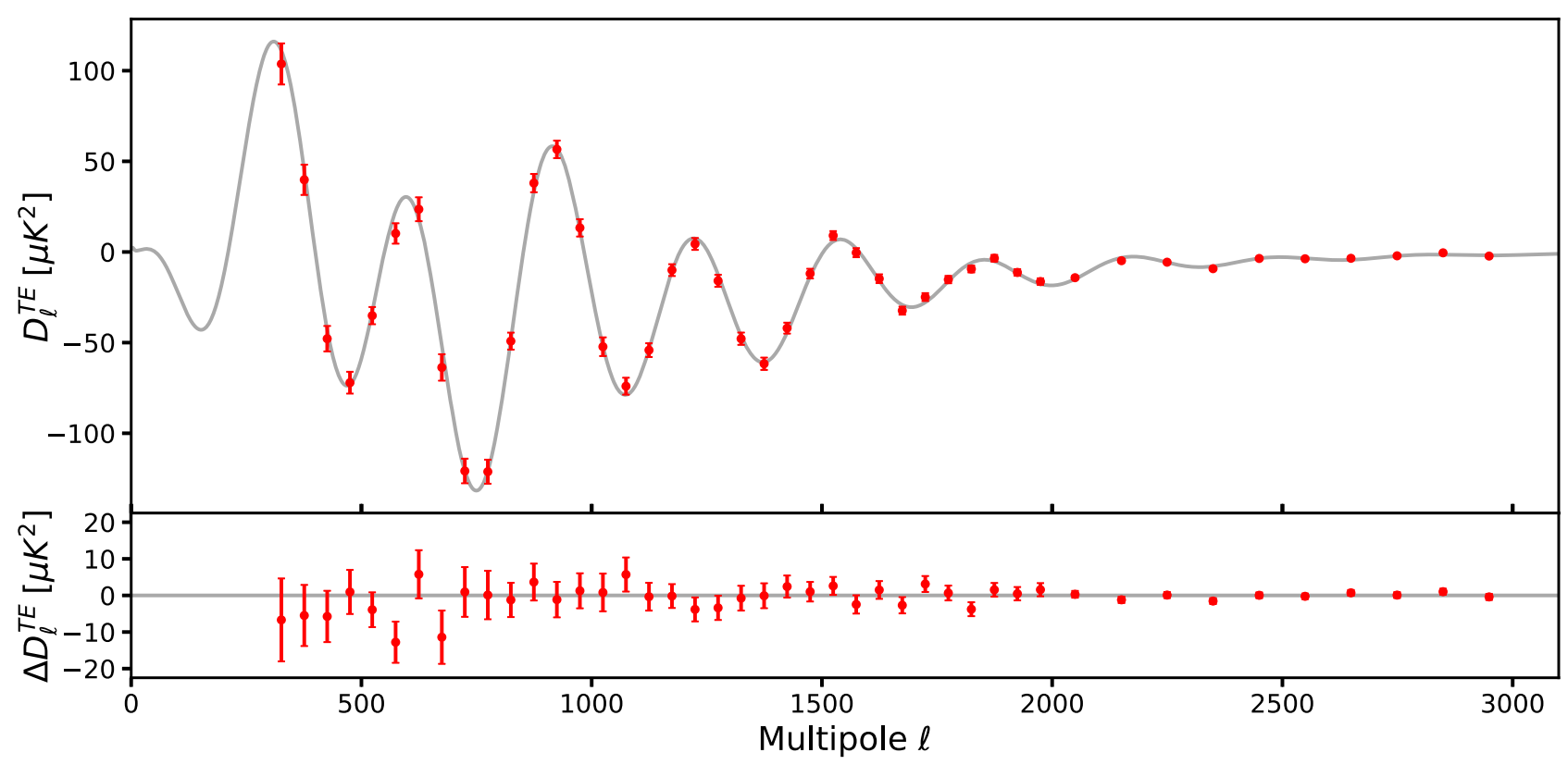

FIG. 11. Minimum-variance $T E$ band powers formed from the six auto- and cross-frequency power spectra and the residuals against the SPT-3G best-fit $\Lambda$ CDM model. Uncertainties are the square root of the diagonal elements of the covariance matrix and do not include beam or calibration uncertainties. 
measurements: it is $1.0 \sigma$ higher than the latest constraints from the Kilo-Degree Survey (KiDS) [70], 0.5 $\sigma$ lower than the Dark Energy Survey (DES) Year 1 results [71] and $0.2 \sigma$ higher than the SZ-selected galaxy cluster measurement from the SPT-SZ survey [72]. This agreement also holds true for the combined growth structure parameter. SPT-3G 2018 infers $S_{8}=\sigma_{8} \sqrt{\Omega_{m} / 0.3}=0.779 \pm 0.041$, which is within $0.3 \sigma, 0.1 \sigma$ and $1.3 \sigma$ of the KiDS, DES, and Planck results, respectively. Adjusting the definition of $S_{8}$ to match the findings of Ref. [72] based on SZ clusters, we find the values to agree within $0.5 \sigma$.

Adding information from BAO measurements [73,74] does not shift the best-fit values of $\Lambda \mathrm{CDM}$ parameters appreciably. However, it tightens the constraint on the density of cold dark matter by a factor of 2.4. This translates into a refined measurement of the Hubble constant of $H_{0}=68.27 \pm 0.63 \mathrm{~km} / \mathrm{s} / \mathrm{Mpc}$, which is comparable to the precision of Planck and disfavors an expansion rate at present day greater than $70 \mathrm{~km} / \mathrm{s} / \mathrm{Mpc}$ at $2.8 \sigma$. The constraints on matter clustering are similarly improved through the inclusion of BAO data by a factor of 1.6 to $0.794 \pm 0.010$ for $\sigma_{8}$ and by a factor of 2.2 for $S_{8}$ to $0.792 \pm 0.018$. The joint SPT-3G and BAO constraint on $\sigma_{8}$ is within $1.2 \sigma$ of the latest result of KiDS, $0.4 \sigma$ of DES, $0.3 \sigma$ of SZ clusters, and $1.5 \sigma$ of Planck. Furthermore, this result is consistent with the joint SPTpol lensing and BAO constraint on $\sigma_{8}$ at $0.6 \sigma$. The joint SPT-3G and BAO constraint on $S_{8}$ is within $1.0 \sigma$ of the latest result of KiDS, $0.6 \sigma$ of DES, $1.0 \sigma$ of SZ clusters, and $1.7 \sigma$ of Planck.

From SPT-3G data alone, we constrain $n_{s}=$ $0.999 \pm 0.019$. While this is slightly higher than the Planck result, a $1.8 \sigma$ offset is not statistically anomalous, especially when analyzed in the context of the full fivedimensional parameter space. Nevertheless, we point out that other ground-based CMB experiments have observed similar trends: the constraints from ACT DR4 [29] and SPTpol $500 \mathrm{deg}^{2} \mathrm{H} 18$ lie $1.1 \sigma$ and $1.3 \sigma$ above the Planck value, respectively, though we expect the SPTpol result to be mildly correlated with ours due to the shared sky area. We explore this facet of the data further in Sec. VIII C.

More generally, our results match those of other contemporary CMB experiments. Given the small shared sky area between SPT-3G 2018 and Planck, we neglect correlations and quantify the difference across the five independent $\Lambda \mathrm{CDM}$ model parameters. We obtain $\chi^{2}=8.8$, which corresponds to a PTE of 0.12 and indicates that the two data sets are consistent.

We confirm that the SPT-3G 2018 data set is consistent with the $\Lambda \mathrm{CDM}$ model by comparing the full set of multifrequency $E E$ and $T E$ band powers to the best-fit $\Lambda \mathrm{CDM}$ model. We quantify the goodness of fit by calculating the associated $\chi^{2}$ over the 528 band powers, finding $\chi^{2}=513.0$. Since nuisance parameters are dominated by their priors, we account for the five free $\Lambda \mathrm{CDM}$ parameters in translating this to the PTE of 0.61 . Comparing the best-fit model to the $E E$ (TE) band powers individually we find $\chi^{2}=273.2(224.2)$. We conclude that the $\Lambda$ CDM model provides a good fit to the SPT-3G 2018 data set. The $E E$ and $T E$ minimum-variance band powers and residuals to the best-fit model are shown in Figs. 10 and 11 , respectively.

\section{B. Gravitational lensing and $A_{L}$}

Our view of the $z=1100$ Universe is distorted by the gravitational lensing of CMB photons due to intervening matter between us and the surface of last scattering. This adds information about the low-redshift Universe and results in a smoothing of the acoustic peaks of the CMB power spectra. The magnitude of this effect is determined by the power spectrum of the lensing potential, which is derived from the six $\Lambda$ CDM parameters in the standard cosmological model. When allowing for a free scaling of the lensing power spectrum, represented by the parameter $A_{L}$ [75], CMB power spectra from Planck have shown a preference for lensing $2.8 \sigma$ beyond the $\Lambda \mathrm{CDM}$ prediction of unity with $A_{L}=1.180 \pm 0.065$ [26]. H18 reported an $A_{L}$ value below unity at $1.4 \sigma$ with $A_{L}=0.81 \pm 0.14$.

Introducing the lensing amplitude as a free parameter in our analysis, the SPT-3G $2018 E E / T E$ data set produces the constraints summarized in Table VII. The core $\Lambda$ CDM model parameters do not shift appreciably, and we report a lensing amplitude of

$$
A_{L}=0.98 \pm 0.12 \text {. }
$$

We conclude that the SPT-3G $2018 E E / T E$ data set is consistent with the level of gravitational lensing expected by the standard model. The reported lensing amplitude falls within $1.5 \sigma$ of the Planck result. It is similar to the findings of $\mathrm{H} 18$, though we expect a mild degree of correlation between that result and ours due to their shared sky area.

TABLE VII. Marginalized $\Lambda \mathrm{CDM}+\mathrm{A}_{L}$ parameter constraints and $68 \%$ errors from SPT-3G. Best-fit values are given in parentheses.

\begin{tabular}{lc}
\hline \hline \multicolumn{2}{c}{ SPT-3G } \\
\hline \multicolumn{2}{c}{ Free } \\
$\Omega_{b} h^{2}$ & $0.02242 \pm 0.00033(0.02242)$ \\
$\Omega_{c} h^{2}$ & $0.1161 \pm 0.0056(0.1165)$ \\
$100 \theta_{\mathrm{MC}}$ & $1.03956 \pm 0.00081(1.03949)$ \\
$10^{9} A_{s} e^{-2 \tau}$ & $1.827 \pm 0.045(1.83)$ \\
$n_{s}$ & $0.995 \pm 0.024(0.993)$ \\
$A_{L}$ & $0.98 \pm 0.12(0.96)$ \\
$\Omega_{\Lambda}$ & Derived \\
$H_{0}$ & $0.701 \pm 0.032(0.699)$ \\
$\sigma_{8}$ & $68.4 \pm 2.3(68.2)$ \\
$S_{8}$ & $0.793 \pm 0.022(0.795)$ \\
Age/Gyr & $0.792 \pm 0.062(0.795)$ \\
\hline \hline
\end{tabular}



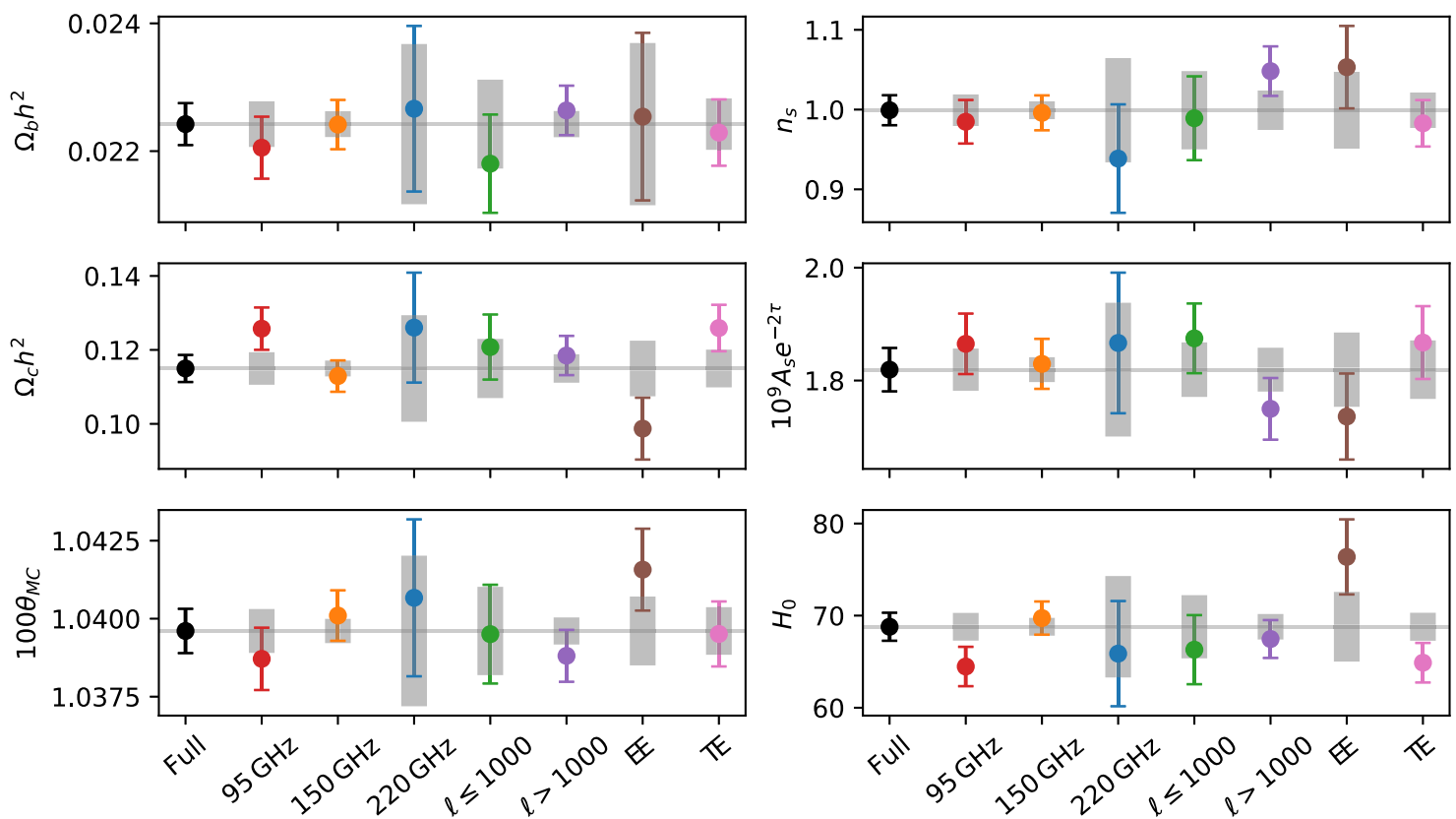

FIG. 12. Parameter constraints from various subsets of the SPT-3G $2018 E E / T E$ data set. The gray boxes correspond to the expected level of statistical fluctuation [65].

\section{Interpretation of data split preferences}

One motivation for studying the CMB polarization anisotropies is that comparing results from the temperature and polarization power spectra yields a stringent test of the $\Lambda \mathrm{CDM}$ cosmological model. Thus while we did not find the parameter differences between subsets of the SPT-3G data to be statistically significant in Sec. VII B, it is still interesting to examine these parameter shifts for possible hints of physics beyond the standard cosmological model. We show the parameter constraints from each data split in Fig. 12. We continue to quantify the significance of parameter shifts as introduced in Sec. VII B, by using the difference of the parameter covariances of the full data set and the given data split.

Examining the best-fit $\Lambda \mathrm{CDM}$ parameters of the different subsets of the SPT-3G $2018 E E / T E$ data set reveals two interesting features. First, the high- $\ell$ data set prefers a scalar spectral index above unity, $n_{s}=1.048 \pm 0.031$, which corresponds to a $2.0 \sigma$ shift from the full data set. With $n_{s}=1.053 \pm 0.052$, the $E E$ spectra prefer a higher scalar spectral index than the high- $\ell$ data set. However, due to their comparatively poor constraining power for this parameter, the $E E$ constraint is only offset by $1.1 \sigma$ from the full data set. The higher value of $n_{s}$ lowers the combined amplitude parameter, as the two are mildly degenerate over the limited $\ell$-range: the high- $\ell$ data prefers $10^{9} A_{s} e^{-2 \tau}=1.750 \pm 0.055$. These values lie $2.0 \sigma$ and $1.8 \sigma$ away from the baseline constraints, respectively. Focusing on the scalar spectral index and the combined amplitude parameter individually, the probability of a shift of the observed size or larger from the full data set constraint is
$2.4 \%$ and $3.7 \%$, respectively. We repeat that fluctuations of this size are statistically not uncommon, especially when viewed in the context of the full five-dimensional parameter space.

A raised scalar spectral index corresponds to a power increase in the damping tail compared to intermediate angular scales. The damping tail is sensitive to an array of

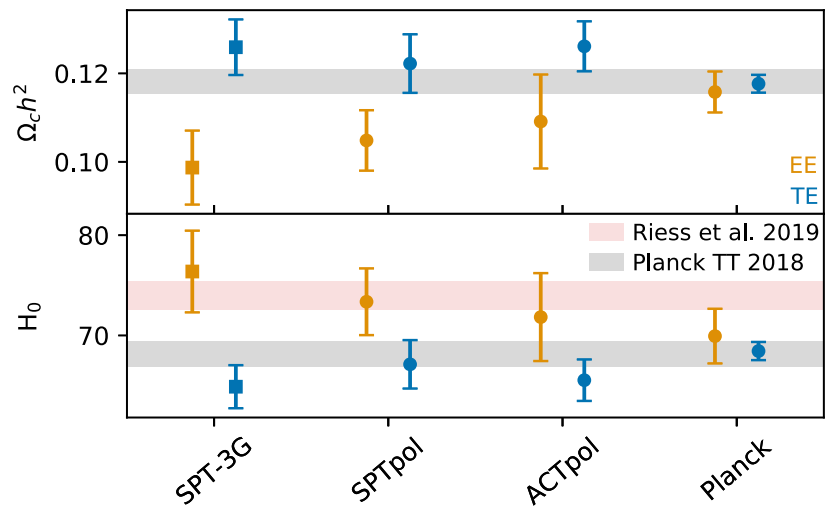

FIG. 13. Constraints on the Hubble constant and cold dark matter density from contemporary CMB experiments. For each experiment, the constraints from $E E$ and $T E$ power spectra are shown in orange and in blue, respectively. The results highlighted here are from this work, H18, Ref. [12] and Ref. [26]. We point out the similarities across experiments, though we note that we expect our results to be mildly correlated with $\mathrm{H} 18$ due to the shared sky area. We also show the $1 \sigma$ constraints on $H_{0}$ from the most recent Cepheid-calibrated distance ladder measurement (red band) [27] and the latest Planck TT-based constraints (gray band) [26] for reference. 


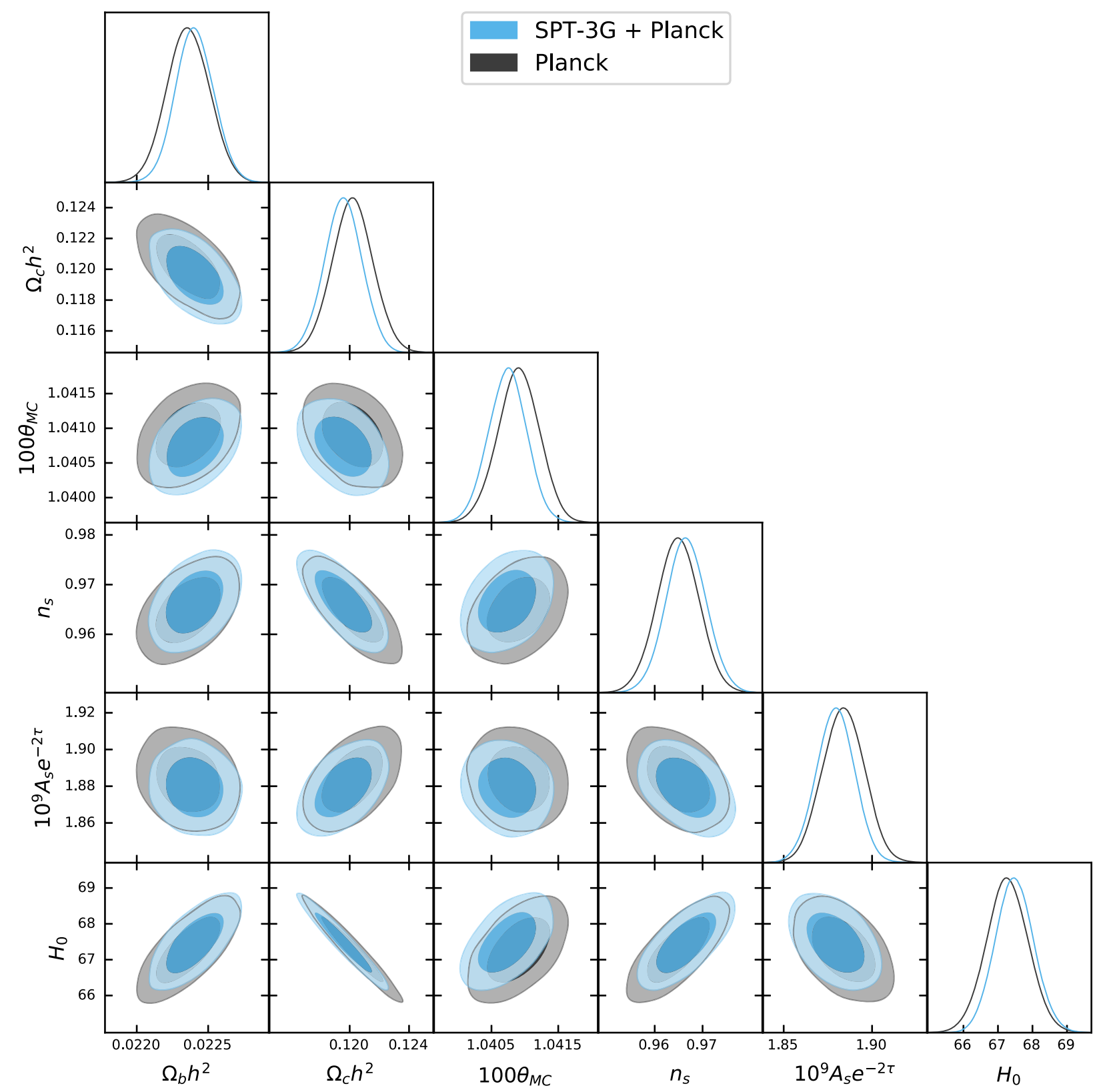

FIG. 14. Joint marginalized constraints on $\Lambda$ CDM parameters and the Hubble constant from the SPT-3G 2018 EE/TE + Planck [1] data sets. Planck-only constraints are shown for comparison.

interesting physics beyond the standard model, such as extra energy injection in the early Universe. This can be explored by allowing the number of relativistic species at recombination, $N_{\text {eff }}$, to vary from the standard model prediction, breaking big bang nucleosynthesis consistency by changing the primordial helium abundance, $Y_{\mathrm{P}}$, or both. We will explore the constraints the SPT-3G 2018 EE/TE data set places on these $\Lambda \mathrm{CDM}$ model extensions in a forthcoming paper.

The second interesting feature of the data splits is a preference in the $E E$ spectra for a lower cold dark matter density, $\Omega_{c} h^{2}=0.0987 \pm 0.0084$, than the $T E$ spectra, $\Omega_{c} h^{2}=0.1259 \pm 0.0063$. These values are $2.2 \sigma$ and $2.1 \sigma$ away from the full data set constraints, respectively. Consequently, different constraints of the Hubble constant are obtained: $H_{0}=76.4 \pm 4.1 \mathrm{~km} / \mathrm{s} / \mathrm{Mpc}$ from the $E E$ spectra and $H_{0}=65.0 \pm 2.1 \mathrm{~km} / \mathrm{s} / \mathrm{Mpc}$ from the $T E$ spectra. Adding BAO information regularizes the matter density fluctuations and consequently the Hubble constant values: $E E$ spectra then prefer $H_{0}=68.7 \pm 1.0 \mathrm{~km} / \mathrm{s} / \mathrm{Mpc}$ and $T E$ spectra $H_{0}=67.82 \pm 0.66 \mathrm{~km} / \mathrm{s} / \mathrm{Mpc}$. While this signals that solutions to the Hubble tension are difficult to 
achieve within the $\Lambda \mathrm{CDM}$ model, model extensions may reconcile the discrepancy between high- and low-redshift probes [76].

A different way of reconciling the matter content inferred by $E E$ and $T E$ spectra, and through this their constraints on the Hubble constant, is by allowing for a free amplitude of the lensing power spectrum. The matter content implies the strength of lensing-induced acoustic-peak smoothing, which results in a mild degeneracy between the matter density and $A_{L}$. This effect was seen in H18, where differences in constraints on cosmological parameters to Planck were alleviated through this model extension. Indeed, we find for SPT-3G 2018 that the $E E$ spectra prefer $A_{L}=$ $0.71_{-0.30}^{+0.32}$ and the $T E$ spectra $A_{L}=0.99_{-0.29}^{+0.30}$, while constraints on $\Omega_{c} h^{2}$ are brought closer together. This is mirrored by the Hubble constant, which is constrained to $H_{0}=68.1 \pm$ $9.3 \mathrm{~km} / \mathrm{s} / \mathrm{Mpc}$ and $H_{0}=64.6 \pm 3.9 \mathrm{~km} / \mathrm{s} / \mathrm{Mpc}$ by the $E E$ and $T E$ spectra, respectively.

Similar trends for low- and high-multipole data as well as $E E$ and $T E$ spectra were reported by Ref. [29] and H18, though we repeat that we expect a mild degree of correlation between $\mathrm{H} 18$ and our results due to the shared sky area. We compile the different Hubble constant measurements in Fig. 13. While the statistical evidence is currently too low, if future polarization measurements amplify this potential tension with cosmological parameters inferred from the temperature anisotropies, these trends may be signs for physics beyond the standard model of cosmology.

\section{SPT-3G + Planck}

The Planck data set provides the most precise measurement of the temperature and polarization anisotropies of the CMB on large angular scales, while the SPT-3G 2018 $E E / T E$ data set provides sensitive information on intermediate and small angular scales. The two data sets thus naturally complement each other, and we may obtain joint constraints by combining them at the likelihood level. Given the small area shared by the two surveys, we expect correlations to be negligible.

We report joint constraints on $\Lambda \mathrm{CDM}$ parameters from the base_plikHM_tTTEEE_lowl_lowe Planck and SPT-3G $2018 E E / T E$ data sets in Table VI. We present the associated 1D and 2D marginalized posteriors in Fig. 14. The inclusion of SPT-3G data does not alter the Planck best-fit values significantly.

We use the determinants of the $\Lambda \mathrm{CDM}$ parameter covariance matrices as a measure of the marginalized parameter-space volume. The ratio of the matrix determinants for SPT-3G 2018 EE/TE combined with Planck to Planck-alone is 0.46 . This corresponds to a reduction of the $68 \%$ confidence region in six-dimensional $\Lambda \mathrm{CDM}$ parameter space by a factor of 1.5 .

\section{CONCLUSION}

In this work we have presented the first results from SPT3G data. Analyzing 2018 data alone, we have produced high-precision measurements of the CMB $E$-mode angular auto-power and temperature- $E$-mode cross-power spectra over the multipole range $300 \leq \ell<3000$. The reported band powers are the first multifrequency $E E$ and $T E$ measurements produced by an instrument on SPT, and they improve upon previous SPT measurements across the multipole ranges $300 \leq \ell \leq 1400$ for $E E$ and $300 \leq \ell \leq$ 1700 for $T E$, resulting in tighter constraints on cosmological parameters.

The SPT-3G 2018 EE/TE data set is consistent with the $\Lambda \mathrm{CDM}$ model. Analyzing constraints from the 95,150 , and $220 \mathrm{GHz}$ auto-frequency spectra, the $\ell<1000$ versus $\ell>$ 1000 data, and the $E E$ and $T E$ spectra individually, we find no signs of significant internal tension.

The constraints on $\Lambda \mathrm{CDM}$ model parameters generally agree with other contemporary $\mathrm{CMB}$ experiments. We report a value of the Hubble constant of $H_{0}=68.8 \pm$ $1.5 \mathrm{~km} / \mathrm{s} / \mathrm{Mpc}$, in line with the CMB-based measurements of Planck and ACT, as well as TRGB-calibrated local distance ladder data. This is in contrast with the higher values found by Cepheid-calibrated distance ladder data and time-delay measurements from gravitationally lensed quasars. However, we note an interesting trend in CMB-based constraints from several experiments, including our own, which have consistently found high values of the Hubble constant when analyzing EE polarization spectra. The current level of tension between polarization- and temperature-based constraints is not statistically significant, but presents an interesting direction for further investigation. The SPT-3G 2018 data set constrains matter-clustering to $\sigma_{8}=0.789 \pm 0.016, S_{8}=0.779 \pm 0.041$, which is consistent with other CMB-based measurements and low-redshift probes.

Expanding the $\Lambda \mathrm{CDM}$ model to allow for a modified amplitude of the lensing power spectrum does not shift parameter constraints appreciably. With $A_{L}=0.98 \pm 0.12$, the SPT-3G 2018 data set is consistent with the standard model prediction.

By combining the SPT-3G 2018 EE/TE and Planck data sets at the likelihood level, we mildly improve the marginalized 1D constraints over Planck data alone. The volume of the $68 \%$ confidence region is reduced by a factor of 1.5 in six-dimensional $\Lambda \mathrm{CDM}$ parameter space.

Last, we note that the high-precision measurements presented in this work use only one half of one observing season of data, which was taken with nearly half the number of currently operating detectors not contributing. With SPT-3G operating at its full capacity since the start of 2019, we now have data from two full observing seasons on disk, with combined map depths $3-4 \times$ deeper than what was used in this analysis. Future SPT-3G results will 
measure the CMB polarization power spectra with exquisite sensitivity on intermediate and small angular scales, constraining physics beyond the standard model with unprecedented precision.

\section{ACKNOWLEDGMENTS}

The South Pole Telescope program is supported by the National Science Foundation (NSF) through Grants No. PLR-1248097 and No. OPP-1852617. Partial support is also provided by the NSF Physics Frontier Center Grant No. PHY-1125897 to the Kavli Institute of Cosmological Physics at the University of Chicago, the Kavli Foundation, and the Gordon and Betty Moore Foundation through Grant No. GBMF\#947 to the University of Chicago. Argonne National Laboratory's work was supported by the U.S. Department of Energy, Office of High Energy Physics, under Contract No. DE-AC02-06CH11357. Work at Fermi National Accelerator Laboratory, a DOE-OS, HEP User Facility managed by the Fermi Research Alliance, LLC, was supported under Contract No. DE-AC0207CH11359. The Cardiff authors acknowledge support from the UK Science and Technologies Facilities Council (STFC). The CU Boulder group acknowledges support from NSF AST-0956135. The IAP authors acknowledge support from the Centre National d'Études Spatiales (CNES). J. V. acknowledges support from the Sloan Foundation. The Melbourne authors acknowledge support from the University of Melbourne and an Australian Research Council Future Fellowship (FT150100074). The McGill authors acknowledge funding from the Natural Sciences and Engineering Research Council of Canada, Canadian Institute for Advanced Research, and the Fonds de recherche du Québec Nature et technologies. N. W. H. acknowledges support from NSF CAREER Grant No. AST-0956135. The UCLA and MSU authors acknowledge support from NSF AST-1716965 and CSSI-1835865. This research was done using resources provided by the Open Science Grid [77,78], which is supported by the National Science Foundation Grant No. 1148698, and the U.S. Department of Energy's Office of Science. This research used resources of the National Energy Research Scientific Computing Center (NERSC), a U.S. Department of Energy Office of Science User Facility operated under Contract No. DE-AC0205CH11231. Some of the results in this paper have been derived using the healpy and HEALPix packages. The data analysis pipeline also uses the scientific PYTHON stack [79-81].

\section{APPENDIX: $E E$ AND TE BAND-POWER TABLES}

The $E E$ and $T E$ band powers from the six sets of crossfrequency power spectra are presented in Tables VIII and IX, respectively. 
TABLE VIII. $E E$ band powers $D_{b}$ for the six cross-frequency power spectra, along with angular multipole range, band-power window-function-weighted multipole $\ell_{\mathrm{eff}}$, and associated uncertainty, $\sigma$. The band powers and errors are quoted in units of $\mu \mathrm{K}^{2}$. The reported uncertainties are the square root of the diagonal elements of the covariance matrix and do not include beam or calibration uncertainties.

\begin{tabular}{|c|c|c|c|c|c|c|c|c|c|c|c|c|c|}
\hline \multirow[b]{2}{*}{$\ell$ Range } & \multirow[b]{2}{*}{$\ell_{\text {eff }}$} & \multicolumn{2}{|c|}{$95 \times 95 \mathrm{GHz}$} & \multicolumn{2}{|c|}{$95 \times 150 \mathrm{GHz}$} & \multicolumn{2}{|c|}{$95 \times 220 \mathrm{GHz}$} & \multicolumn{2}{|c|}{$150 \times 150 \mathrm{GHz}$} & \multicolumn{2}{|c|}{$150 \times 220 \mathrm{GHz}$} & \multicolumn{2}{|c|}{$220 \times 220 \mathrm{GHz}$} \\
\hline & & $D_{b}$ & $\sigma$ & $D_{b}$ & $\sigma$ & $D_{b}$ & $\sigma$ & $D_{b}$ & $\sigma$ & $D_{b}$ & $\sigma$ & $D_{b}$ & $\sigma$ \\
\hline $300-349$ & 325 & 13.1 & 1.1 & 12.8 & 1.1 & 12.1 & 1.3 & 13.2 & 1.1 & 12.7 & 1.3 & 12.1 & 2.0 \\
\hline $350-399$ & 375 & 19.7 & 1.3 & 20.5 & 1.3 & 19.0 & 1.5 & 21.1 & 1.3 & 19.9 & 1.5 & 18.0 & 2.3 \\
\hline $400-449$ & 425 & 19.0 & 1.2 & 18.8 & 1.1 & 17.9 & 1.3 & 19.1 & 1.1 & 18.4 & 1.3 & 17.7 & 2.1 \\
\hline $450-499$ & 475 & 11.2 & 0.7 & 12.0 & 0.7 & 11.1 & 0.9 & 12.5 & 0.7 & 11.1 & 0.9 & 9.4 & 1.7 \\
\hline $500-549$ & 524 & 7.1 & 0.5 & 7.3 & 0.4 & 7.6 & 0.7 & 7.0 & 0.4 & 8.3 & 0.6 & 9.4 & 1.6 \\
\hline $550-599$ & 575 & 11.1 & 0.7 & 11.3 & 0.6 & 12.2 & 0.9 & 11.8 & 0.7 & 11.8 & 0.9 & 11.5 & 1.9 \\
\hline $600-649$ & 624 & 29.0 & 1.3 & 29.4 & 1.2 & 29.1 & 1.5 & 30.1 & 1.2 & 29.8 & 1.4 & 34.3 & 2.6 \\
\hline $650-699$ & 674 & 39.0 & 1.5 & 39.1 & 1.3 & 39.5 & 1.7 & 38.9 & 1.4 & 39.7 & 1.7 & 40.9 & 2.9 \\
\hline $700-749$ & 725 & 33.6 & 1.4 & 34.4 & 1.3 & 33.1 & 1.7 & 35.0 & 1.3 & 34.1 & 1.6 & 32.4 & 3.0 \\
\hline $750-799$ & 774 & 21.2 & 1.1 & 20.8 & 0.9 & 22.0 & 1.3 & 20.4 & 0.9 & 21.3 & 1.2 & 22.8 & 2.7 \\
\hline 800-849 & 824 & 13.2 & 0.8 & 13.3 & 0.6 & 13.2 & 1.0 & 13.7 & 0.6 & 13.4 & 0.9 & 13.6 & 2.6 \\
\hline 850-899 & 874 & 16.9 & 0.9 & 17.2 & 0.7 & 17.8 & 1.2 & 17.0 & 0.8 & 17.7 & 1.1 & 19.1 & 2.9 \\
\hline 900-949 & 924 & 31.8 & 1.3 & 31.4 & 1.1 & 30.8 & 1.7 & 31.6 & 1.1 & 32.3 & 1.6 & 29.6 & 3.5 \\
\hline 950-999 & 974 & 41.2 & 1.6 & 40.4 & 1.4 & 40.7 & 2.0 & 40.7 & 1.4 & 39.9 & 1.9 & 36.9 & 4.0 \\
\hline 1000-1049 & 1024 & 39.4 & 1.6 & 38.4 & 1.3 & 39.3 & 2.0 & 38.5 & 1.4 & 37.3 & 1.9 & 40.7 & 4.2 \\
\hline 1050-1099 & 1075 & 26.1 & 1.3 & 26.3 & 1.0 & 24.9 & 1.7 & 26.4 & 1.1 & 25.3 & 1.6 & 20.4 & 4.0 \\
\hline 1100-1149 & 1124 & 15.5 & 1.0 & 15.2 & 0.7 & 14.6 & 1.4 & 15.0 & 0.7 & 13.9 & 1.2 & 10.7 & 3.9 \\
\hline 1150-1199 & 1174 & 13.1 & 1.0 & 12.3 & 0.7 & 10.8 & 1.5 & 12.6 & 0.7 & 12.1 & 1.2 & 12.6 & 4.1 \\
\hline $1200-1249$ & 1224 & 20.6 & 1.3 & 21.8 & 0.9 & 23.9 & 1.8 & 22.1 & 1.0 & 22.3 & 1.6 & 18.0 & 4.6 \\
\hline 1250-1299 & 1275 & 29.9 & 1.5 & 29.2 & 1.1 & 28.5 & 2.1 & 29.6 & 1.2 & 26.9 & 1.9 & 26.9 & 5.2 \\
\hline 1300-1349 & 1325 & 31.2 & 1.6 & 30.9 & 1.1 & 28.5 & 2.2 & 32.1 & 1.2 & 28.5 & 1.9 & 24.4 & 5.5 \\
\hline 1350-1399 & 1374 & 24.1 & 1.4 & 22.4 & 1.0 & 22.2 & 2.1 & 22.2 & 1.0 & 25.0 & 1.8 & 40.0 & 5.7 \\
\hline 1400-1449 & 1424 & 14.1 & 1.3 & 13.0 & 0.8 & 11.9 & 1.9 & 12.6 & 0.8 & 11.3 & 1.6 & 5.5 & 5.9 \\
\hline 1450-1499 & 1474 & 10.9 & 1.3 & 10.2 & 0.7 & 11.4 & 2.0 & 10.4 & 0.8 & 13.4 & 1.6 & 19.2 & 6.2 \\
\hline $1500-1549$ & 1524 & 15.0 & 1.4 & 15.4 & 0.8 & 12.6 & 2.2 & 14.1 & 0.9 & 11.1 & 1.8 & 8.0 & 6.7 \\
\hline 1550-1599 & 1574 & 22.1 & 1.6 & 20.9 & 1.0 & 22.1 & 2.4 & 21.1 & 1.0 & 24.1 & 2.0 & 23.8 & 7.2 \\
\hline 1600-1649 & 1624 & 17.6 & 1.7 & 20.0 & 1.1 & 20.4 & 2.6 & 20.7 & 1.1 & 21.7 & 2.1 & 24.0 & 7.6 \\
\hline 1650-1699 & 1674 & 19.2 & 1.7 & 18.4 & 1.0 & 14.7 & 2.6 & 18.1 & 1.0 & 18.9 & 2.0 & 12.9 & 8.0 \\
\hline 1700-1749 & 1724 & 7.4 & 1.7 & 10.2 & 0.9 & 10.8 & 2.6 & 10.6 & 0.9 & 14.2 & 2.0 & 0.3 & 8.3 \\
\hline 1750-1799 & 1775 & 10.1 & 1.7 & 8.7 & 0.9 & 11.3 & 2.7 & 8.5 & 0.9 & 8.0 & 2.0 & 14.9 & 8.8 \\
\hline 1800-1849 & 1825 & 8.3 & 1.8 & 9.0 & 0.9 & 5.8 & 2.9 & 9.6 & 0.9 & 5.4 & 2.1 & -0.4 & 9.4 \\
\hline 1850-1899 & 1874 & 9.7 & 2.0 & 9.8 & 1.0 & 9.6 & 3.2 & 9.8 & 1.0 & 13.1 & 2.3 & 14.2 & 10.0 \\
\hline 1900-1949 & 1924 & 12.7 & 2.1 & 12.9 & 1.1 & 18.2 & 3.3 & 12.0 & 1.1 & 7.8 & 2.4 & 0.6 & 10.6 \\
\hline 1950-1999 & 1975 & 12.4 & 2.2 & 10.2 & 1.1 & 8.9 & 3.5 & 11.4 & 1.1 & 13.9 & 2.5 & 6.2 & 11.2 \\
\hline 2000-2099 & 2049 & 6.7 & 1.2 & 6.3 & 0.6 & 7.9 & 2.0 & 6.3 & 0.6 & 6.2 & 1.4 & 4.9 & 6.7 \\
\hline 2100-2199 & 2148 & 5.3 & 1.4 & 5.6 & 0.7 & 1.1 & 2.3 & 5.4 & 0.7 & 5.4 & 1.6 & 9.0 & 7.6 \\
\hline 2200-2299 & 2248 & 7.3 & 1.6 & 7.6 & 0.8 & 6.8 & 2.6 & 6.0 & 0.7 & 7.2 & 1.8 & 8.7 & 8.6 \\
\hline 2300-2399 & 2348 & 1.2 & 1.8 & 2.6 & 0.8 & 4.2 & 2.9 & 4.9 & 0.8 & 1.0 & 1.9 & 13.3 & 9.4 \\
\hline 2400-2499 & 2448 & 6.8 & 2.0 & 4.0 & 0.9 & 5.2 & 3.2 & 2.6 & 0.8 & 5.2 & 2.1 & -0.8 & 10.4 \\
\hline $2500-2599$ & 2548 & 2.9 & 2.2 & 2.5 & 1.0 & 0.2 & 3.5 & 2.6 & 0.9 & 3.0 & 2.3 & -2.5 & 11.5 \\
\hline $2600-2699$ & 2648 & 5.9 & 2.5 & 0.5 & 1.1 & -0.1 & 4.0 & 2.3 & 1.0 & 2.1 & 2.5 & 10.5 & 12.6 \\
\hline 2700-2799 & 2748 & -0.9 & 2.8 & 0.8 & 1.3 & 9.5 & 4.5 & 2.0 & 1.1 & 3.4 & 2.8 & -6.4 & 14.1 \\
\hline $2800-2899$ & 2848 & 0.6 & 3.2 & 3.0 & 1.4 & 4.5 & 5.0 & 0.5 & 1.3 & -3.2 & 3.2 & -5.8 & 15.7 \\
\hline 2900-2999 & 2948 & -1.2 & 3.6 & -2.4 & 1.6 & -7.2 & 5.6 & 1.0 & 1.4 & 7.4 & 3.5 & -3.6 & 17.1 \\
\hline
\end{tabular}


TABLE IX. TE band powers $D_{b}$ for the six cross-frequency power spectra, along with angular multipole range, band-power windowfunction-weighted multipole $\ell_{\text {eff }}$, and associated uncertainty, $\sigma$. The band powers and errors are quoted in units of $\mu \mathrm{K}^{2}$. The reported uncertainties are the square root of the diagonal elements of the covariance matrix and do not include beam or calibration uncertainties.

\begin{tabular}{|c|c|c|c|c|c|c|c|c|c|c|c|c|c|}
\hline \multirow[b]{2}{*}{$\ell$ Range } & \multirow[b]{2}{*}{$\ell_{\text {eff }}$} & \multicolumn{2}{|c|}{$95 \times 95 \mathrm{GHz}$} & \multicolumn{2}{|c|}{$95 \times 150 \mathrm{GHz}$} & \multicolumn{2}{|c|}{$95 \times 220 \mathrm{GHz}$} & \multicolumn{2}{|c|}{$150 \times 150 \mathrm{GHz}$} & \multicolumn{2}{|c|}{$150 \times 220 \mathrm{GHz}$} & \multicolumn{2}{|c|}{$220 \times 220 \mathrm{GHz}$} \\
\hline & & $D_{b}$ & $\sigma$ & $D_{b}$ & $\sigma$ & $D_{b}$ & $\sigma$ & $D_{b}$ & $\sigma$ & $D_{b}$ & $\sigma$ & $D_{b}$ & $\sigma$ \\
\hline $300-349$ & 326 & 88.4 & 12.0 & 93.2 & 12.1 & 99.8 & 13.7 & 101.1 & 12.7 & 110.5 & 14.0 & 113.7 & 20.3 \\
\hline $350-399$ & 376 & 43.6 & 8.8 & 42.4 & 8.7 & 36.6 & 10.5 & 42.7 & 9.2 & 40.8 & 10.7 & 40.1 & 17.2 \\
\hline $400-449$ & 426 & -44.7 & 7.6 & -45.6 & 7.3 & -43.0 & 9.0 & -47.8 & 7.5 & -47.1 & 9.0 & -43.4 & 15.0 \\
\hline $450-499$ & 475 & -68.8 & 6.7 & -68.9 & 6.2 & -65.0 & 7.8 & -70.0 & 6.4 & -64.5 & 7.7 & -53.2 & 13.2 \\
\hline $500-549$ & 523 & -34.0 & 5.5 & -34.6 & 5.0 & -48.2 & 6.7 & -34.8 & 5.2 & -46.7 & 6.7 & -58.2 & 12.2 \\
\hline $550-599$ & 574 & 11.8 & 6.2 & 11.2 & 5.8 & 15.2 & 7.4 & 10.5 & 6.1 & 15.6 & 7.3 & 20.8 & 12.4 \\
\hline $600-649$ & 625 & 24.1 & 7.0 & 23.8 & 6.7 & 21.5 & 8.1 & 24.5 & 7.0 & 23.1 & 8.1 & 21.4 & 12.8 \\
\hline $650-699$ & 675 & -63.3 & 7.7 & -63.3 & 7.4 & -58.0 & 8.7 & -63.1 & 7.5 & -59.2 & 8.6 & -60.0 & 13.0 \\
\hline $700-749$ & 725 & -119.5 & 7.3 & -120.9 & 6.9 & -114.0 & 8.2 & -122.8 & 7.0 & -116.0 & 8.1 & -105.2 & 12.7 \\
\hline 750-799 & 774 & -121.2 & 7.2 & -120.4 & 6.7 & -124.1 & 8.3 & -121.3 & 6.8 & -126.2 & 8.1 & -124.6 & 12.9 \\
\hline 800-849 & 824 & -52.6 & 5.6 & -50.5 & 4.8 & -43.2 & 6.8 & -48.6 & 5.0 & -40.0 & 6.7 & -25.6 & 12.1 \\
\hline 850-899 & 874 & 41.0 & 5.8 & 38.5 & 5.1 & 38.5 & 6.9 & 36.6 & 5.3 & 37.2 & 6.8 & 36.7 & 11.9 \\
\hline 900-949 & 924 & 54.5 & 5.5 & 56.0 & 4.9 & 58.9 & 6.6 & 56.9 & 5.1 & 61.5 & 6.5 & 70.4 & 11.3 \\
\hline 950-999 & 974 & 12.4 & 5.3 & 13.1 & 4.8 & 14.4 & 6.3 & 13.9 & 5.0 & 13.8 & 6.2 & 18.0 & 10.6 \\
\hline 1000-1049 & 1024 & -52.0 & 5.6 & -51.8 & 5.2 & -55.5 & 6.5 & -51.7 & 5.4 & -55.8 & 6.4 & -56.7 & 10.6 \\
\hline 1050-1099 & 1075 & -75.6 & 5.3 & -74.6 & 4.7 & -71.9 & 6.2 & -73.7 & 4.9 & -72.1 & 6.1 & -70.1 & 10.4 \\
\hline 1100-1149 & 1124 & -48.3 & 4.6 & -52.7 & 3.9 & -58.4 & 5.6 & -55.9 & 4.1 & -60.3 & 5.5 & -66.0 & 10.2 \\
\hline 1150-1199 & 1174 & -9.7 & 4.2 & -10.1 & 3.4 & -6.9 & 5.3 & -10.8 & 3.6 & -7.1 & 5.1 & -1.9 & 10.0 \\
\hline $1200-1249$ & 1224 & 4.9 & 4.1 & 4.3 & 3.4 & 4.2 & 5.1 & 4.3 & 3.6 & 4.3 & 5.0 & 8.3 & 9.8 \\
\hline $1250-1299$ & 1274 & -15.4 & 4.1 & -15.7 & 3.4 & -17.2 & 5.0 & -16.0 & 3.6 & -16.7 & 4.9 & -16.4 & 9.6 \\
\hline 1300-1349 & 1324 & -47.1 & 4.2 & -48.1 & 3.5 & -43.6 & 5.1 & -49.1 & 3.7 & -42.9 & 4.9 & -39.7 & 9.6 \\
\hline 1350-1399 & 1374 & -61.8 & 4.3 & -61.8 & 3.5 & -55.3 & 5.3 & -63.0 & 3.7 & -56.8 & 5.1 & -47.5 & 10.0 \\
\hline $1400-1449$ & 1424 & -41.0 & 4.1 & -41.8 & 3.1 & -41.2 & 5.2 & -42.8 & 3.3 & -41.1 & 5.0 & -30.8 & 10.2 \\
\hline 1450-1499 & 1474 & -10.9 & 3.8 & -11.8 & 2.8 & -8.6 & 5.0 & -13.0 & 3.0 & -9.9 & 4.8 & -4.2 & 10.1 \\
\hline $1500-1549$ & 1524 & 8.4 & 3.6 & 9.0 & 2.6 & 4.8 & 4.7 & 10.2 & 2.8 & 5.9 & 4.5 & -7.4 & 9.8 \\
\hline 1550-1599 & 1574 & -3.8 & 3.5 & -0.8 & 2.6 & -4.2 & 4.5 & 1.1 & 2.8 & 0.3 & 4.3 & -5.1 & 9.5 \\
\hline $1600-1649$ & 1624 & -13.9 & 3.4 & -15.4 & 2.5 & -15.8 & 4.3 & -14.5 & 2.7 & -13.3 & 4.1 & -8.0 & 9.4 \\
\hline 1650-1699 & 1674 & -31.0 & 3.3 & -32.0 & 2.4 & -32.4 & 4.3 & -33.1 & 2.5 & -31.7 & 4.0 & -33.1 & 9.5 \\
\hline 1700-1749 & 1724 & -21.9 & 3.3 & -24.0 & 2.3 & -25.9 & 4.4 & -25.9 & 2.5 & -26.7 & 4.1 & -25.1 & 9.8 \\
\hline 1750-1799 & 1775 & -15.7 & 3.3 & -15.1 & 2.2 & -17.6 & 4.4 & -14.7 & 2.4 & -17.4 & 4.1 & -21.5 & 10.0 \\
\hline 1800-1849 & 1824 & -14.1 & 3.2 & -10.0 & 2.1 & -7.1 & 4.3 & -8.4 & 2.2 & -7.3 & 3.9 & 3.4 & 9.9 \\
\hline 1850-1899 & 1874 & -3.8 & 3.0 & -3.3 & 2.0 & -5.1 & 4.1 & -3.4 & 2.2 & -3.3 & 3.8 & -12.6 & 9.8 \\
\hline 1900-1949 & 1924 & -11.8 & 3.0 & -11.2 & 2.0 & -10.8 & 4.1 & -11.3 & 2.2 & -11.0 & 3.7 & -14.0 & 9.8 \\
\hline 1950-1999 & 1975 & -15.0 & 3.0 & -16.4 & 2.0 & -17.8 & 4.1 & -16.3 & 2.1 & -17.3 & 3.7 & -18.7 & 10.1 \\
\hline 2000-2099 & 2050 & -16.0 & 1.7 & -14.2 & 1.0 & -14.6 & 2.3 & -13.8 & 1.1 & -14.0 & 2.1 & -17.6 & 5.8 \\
\hline 2100-2199 & 2151 & -5.4 & 1.6 & -4.7 & 1.0 & -9.1 & 2.3 & -4.3 & 1.1 & -5.8 & 2.1 & 3.7 & 6.1 \\
\hline $2200-2299$ & 2250 & -7.6 & 1.6 & -6.3 & 1.0 & -3.9 & 2.3 & -5.0 & 1.0 & -3.6 & 2.0 & -9.2 & 6.4 \\
\hline $2300-2399$ & 2349 & -8.9 & 1.6 & -8.8 & 1.0 & -10.6 & 2.4 & -9.3 & 1.0 & -10.5 & 2.0 & -19.6 & 6.7 \\
\hline 2400-2499 & 2450 & -7.4 & 1.7 & -4.7 & 0.9 & -5.8 & 2.4 & -2.3 & 1.0 & -0.4 & 2.0 & 0.1 & 7.0 \\
\hline $2500-2599$ & 2549 & -0.9 & 1.7 & -4.2 & 0.9 & -4.0 & 2.5 & -3.6 & 1.0 & -5.1 & 2.0 & -14.3 & 7.4 \\
\hline $2600-2699$ & 2649 & -5.0 & 1.8 & -3.3 & 1.0 & -6.5 & 2.7 & -3.2 & 1.0 & -3.5 & 2.1 & -2.0 & 7.9 \\
\hline 2700-2799 & 2749 & 1.5 & 1.9 & -2.1 & 1.0 & 5.5 & 2.9 & -3.8 & 1.0 & 1.9 & 2.2 & 16.3 & 8.5 \\
\hline 2800-2899 & 2849 & 2.4 & 2.1 & 0.2 & 1.1 & -0.3 & 3.1 & -0.7 & 1.0 & -5.5 & 2.3 & -3.6 & 9.2 \\
\hline 2900-2999 & 2949 & -6.9 & 2.3 & -1.8 & 1.1 & -5.3 & 3.3 & -2.1 & 1.1 & 0.2 & 2.4 & 15.6 & 9.7 \\
\hline
\end{tabular}


[1] N. Aghanim, Y. Akrami et al. (Planck Collaboration), Planck 2018 results. V. CMB power spectra and likelihoods, Astropart. Phys. 641, A5 (2020).

[2] T. Louis, E. Grace, M. Hasselfield et al., The atacama cosmology telescope: Two-season ACTPol spectra and parameters, J. Cosmol. Astropart. Phys. 6 (2017) 031.

[3] C. L. Reichardt, S. Patil, P. A. R. Ade et al., An improved measurement of the secondary cosmic microwave background anisotropies from the SPT-SZ + SPTpol surveys, arXiv:2002.06197.

[4] W. Hu and M. White, A CMB polarization primer, New Astron. 2, 323 (1997).

[5] U. Seljak and M. Zaldarriaga, Signature of Gravity Waves in the Polarization of the Microwave Background, Phys. Rev. Lett. 78, 2054 (1997).

[6] M. Kamionkowski, A. Kosowsky, and A. Stebbins, A Probe of Primordial Gravity Waves and Vorticity, Phys. Rev. Lett. 78, 2058 (1997).

[7] L. Knox and Y. Song, Limit on the Detectability of the Energy Scale of Inflation, Phys. Rev. Lett. 89, 011303 (2002).

[8] S. Galli, K. Benabed, F. Bouchet, J.-F. Cardoso, F. Elsner, E. Hivon, A. Mangilli, S. Prunet, and B. Wandelt, CMB polarization can constrain cosmology better than CMB temperature, Phys. Rev. D 90, 063504 (2014).

[9] N. Gupta, C. L. Reichardt, P. A. R. Ade et al., Fractional polarization of extragalactic sources in the $500 \mathrm{deg}^{2}$ SPTpol survey, Mon. Not. R. Astron. Soc. 490, 5712 (2019).

[10] R. Datta, S. Aiola, S. K. Choi et al., The atacama cosmology telescope: Two-season ACTPol extragalactic point sources and their polarization properties, Mon. Not. R. Astron. Soc. 486, 5239 (2019).

[11] T. Trombetti, C. Burigana, G. De Zotti, V. Galluzzi, and M. Massardi, Average fractional polarization of extragalactic sources at Planck frequencies, Astropart. Phys. 618, A29 (2018).

[12] S. K. Choi, M. Hasselfield, S.-P. P. Ho et al., The atacama cosmology telescope: A measurement of the cosmic microwave background power spectra at 98 and $150 \mathrm{GHz}$, arXiv:2007.07289.

[13] P. A. R. Ade et al. (BICEP2 Collaboration and Keck Array Collaboration), Constraints on Primordial Gravitational Waves Using Planck, WMAP, and New BICEP2/Keck Observations through the 2015 Season, Phys. Rev. Lett. 121, 221301 (2018).

[14] S. Adachi and M. A. O. Aguilar Faúndez et al. (POLARBEAR Collaboration), A measurement of the degree scale CMB B-mode angular power spectrum with POLARBEAR, arXiv:1910.02608.

[15] S. Adachi, M. A. O. Aguilar Faúndez, K. Arnold et al., A measurement of the CMB E-mode angular power spectrum at subdegree scales from 670 square degrees of POLARBEAR data, arXiv:2005.06168.

[16] J. W. Henning, J. T. Sayre, C. L. Reichardt et al., Measurements of the temperature and E-mode polarization of the CMB from 500 square degrees of SPTpol data, Astrophys. J. 852, 97 (2018).

[17] J. T. Sayre, C. L. Reichardt, J. W. Henning et al., Measurements of B-mode polarization of the cosmic microwave background from 500 square degrees of SPTpol data, Phys. Rev. D 101, 122003 (2020).
[18] S. W. Henderson, R. Allison, J. Austermann et al., Advanced ACTPol cryogenic detector arrays and readout, J. Low Temp. Phys. 184, 772 (2016).

[19] Z. Ahmed, M. Amiri, S. J. Benton et al., BICEP3: A 95 GHz refracting telescope for degree-scale $\mathrm{CMB}$ polarization, in Proc. SPIE, Society of Photo-Optical Instrumentation Engineers (SPIE) Conference Series Vol. 9153 (SPIE, 2014), p. 1, https://doi.org/10.1117/12.2057224.

[20] H. Hui, P. A. R. Ade, Z. Ahmed et al., BICEP Array: A multi-frequency degree-scale CMB polarimeter, in Proc. SPIE Society of Photo-Optical Instrumentation Engineers (SPIE) Conference Series Vol. 10708 (SPIE, 2018), p. 1070807, https://doi.org/10.1117/12.2311725.

[21] A. Suzuki, P. Ade, Y. Akiba et al., The POLARBEAR-2 and the Simons Array Experiments, J. Low Temp. Phys. 184, 805 (2016).

[22] P. Ade, J. Aguirre et al. (Simons Observatory Collaboration), The simons observatory: Science goals and forecasts, J. Cosmol. Astropart. Phys. 02 (2019) 056.

[23] B. A. Benson, P. A. R. Ade, Z. Ahmed et al., SPT-3G: A nextgeneration cosmic microwave background polarization experiment on the south pole telescope, in Proc. SPIE, Millimeter, Submillimeter, and Far-Infrared Detectors and Instrumentation for Astronomy VII Vol. 9153 (SPIE, 2014), p. 91531P, https:// doi.org/10.1117/12.2057305.

[24] K. Aylor, Z. Hou, L. Knox et al., A comparison of cosmological parameters determined from CMB temperature power spectra from the south pole telescope and the planck satellite, Astrophys. J. 850, 101 (2017).

[25] G. E. Addison, Y. Huang, D. J. Watts, C. L. Bennett, M. Halpern, G. Hinshaw, and J. L. Weiland, Quantifying discordance in the 2015 planck CMB spectrum, Astrophys. J. 818, 132 (2016).

[26] N. Aghanim, Y. Akrami et al. (Planck Collaboration), Planck 2018 results. VI. Cosmological parameters, Astropart. Phys. 641, A6 (2020).

[27] A. G. Riess, S. Casertano, W. Yuan, L. M. Macri, and D. Scolnic, Large magellanic cloud cepheid standards provide a $1 \%$ foundation for the determination of the Hubble constant and stronger evidence for physics beyond

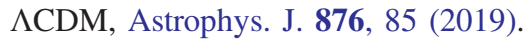

[28] J. E. Carlstrom, P. A. R. Ade, K. A. Aird et al., The 10 Meter south pole telescope, Publ. Astron. Soc. Pac. 123, 568 (2011).

[29] S. Aiola, E. Calabrese, L. Maurin et al., The atacama cosmology telescope: DR4 maps and cosmological parameters, arXiv:2007.07288.

[30] J. A. Sobrin, P. A. R. Ade, Z. Ahmed et al., Design and characterization of the SPT-3G receiver, in Proc. SPIE (SPIE, 2018), Vol. 10708, p. 107081H, https://doi.org/10.1117/ 12.2314366 .

[31] A. Nadolski, J. D. Vieira, J. A. Sobrin et al., Broadband, millimeter-wave antireflection coatings for large-format, cryogenic aluminum oxide optics, Appl. Opt. 59, 3285 (2020).

[32] N. Galitzki, A. Ali, K. S. Arnold et al., The simons observatory: Instrument overview, in Proc. SPIE (SPIE, 2018), Vol. 10708, p. 1070804, https://doi.org/10.1117/ 12.2312985 .

[33] A. Suzuki, P. A. R. Ade, Y. Akiba et al., The LiteBIRD satellite mission: Sub-Kelvin instrument, J. Low Temp. Phys. 193, 1048 (2018). 
[34] C. M. Posada, P. A. R. Ade, Z. Ahmed et al., Fabrication of large dual-polarized multichroic TES bolometer arrays for CMB measurements with the SPT-3G camera, Supercond. Sci. Technol. 28, 094002 (2015).

[35] C. M. Posada, Fabrication of detector arrays for the SPT-3G receiver, J. Low Temp. Phys. 193, 703 (2018).

[36] D. Dutcher, P. A. R. Ade, Z. Ahmed et al., Characterization and performance of the second-year SPT-3G focal plane, in Proc. SPIE (SPIE, 2018), Vol. 10708, p. 107081Z, https:// doi.org/10.1117/12.2312451.

[37] A. N. Bender, J.-F. Cliche, T. de Haan, M. A. Dobbs, A. J. Gilbert, J. Montgomery, N. Rowlands, G. M. Smecher, K. Smith, and A. Wilson, Digital frequency domain multiplexing readout electronics for the next generation of millimeter telescopes, in Proc. SPIE, Millimeter, Submillimeter, and Far-Infrared Detectors and Instrumentation for Astronomy VII Vol. 9153 (SPIE, 2014), p. 91531A, https://doi.org/10.1117/12.2054949.

[38] A. N. Bender, P. A. R. Ade, A. J. Anderson et al., Integrated performance of a frequency domain multiplexing readout in the SPT-3G receiver, in Proc. SPIE, Millimeter, Submillimeter, and Far-Infrared Detectors and Instrumentation for Astronomy VIII Vol. 9914 (SPIE, 2016), p. 99141D, https:// doi.org/10.1117/12.2232146.

[39] R. Adam, P. A. R. Ade et al. (Planck Collaboration), Planck 2015 results. X. Diffuse component separation: Foreground maps, Astropart. Phys. 594, A10 (2016).

[40] K. T. Story, C. L. Reichardt, Z. Hou et al., A measurement of the cosmic microwave background damping tail from the 2500-Square-Degree SPT-SZ survey, Astrophys. J. 779, 86 (2013).

[41] A. T. Crites, J. W. Henning, P. A. R. Ade et al., Measurements of E-mode polarization and temperature-E-mode correlation in the cosmic microwave background from 100 Square Degrees of SPTpol data, Astrophys. J. 805, 36 (2015).

[42] R. Keisler, S. Hoover, N. Harrington et al., Measurements of Sub-degree B-mode polarization in the cosmic microwave background from 100 Square Degrees of SPTpol data, Astrophys. J. 807, 151 (2015).

[43] W. C. Jones, T. E. Montroy, B. P. Crill, C. R. Contaldi, T. S. Kisner, A. E. Lange, C. J. MacTavish, C. B. Netterfield, and J. E. Ruhl, Instrumental and analytic methods for bolometric polarimetry, Astropart. Phys. 470, 771 (2007).

[44] M. Zaldarriaga, Nature of the E-B decomposition of CMB polarization, Phys. Rev. D 64, 103001 (2001).

[45] M. Tristram, J. F. Macías-Pérez, C. Renault, and D. Santos, XSPECT, estimation of the angular power spectrum by computing cross-power spectra with analytical error bars, Mon. Not. R. Astron. Soc. 358, 833 (2005).

[46] G. Polenta, D. Marinucci, A. Balbi, P. de Bernardis, E. Hivon, S. Masi, P. Natoli, and N. Vittorio, Unbiased estimation of an angular power spectrum, J. Cosmol. Astropart. Phys. 11 (2005) 001.

[47] E. Hivon, K. M. Górski, C. B. Netterfield, B. P. Crill, S. Prunet, and F. Hansen, MASTER of the cosmic microwave background anisotropy power spectrum: A fast method for statistical analysis of large and complex cosmic microwave background data sets, Astrophys. J. 567, 2 (2002).
[48] K. M. Górski, E. Hivon, A. J. Banday, B. D. Wandelt, F. K. Hansen, M. Reinecke, and M. Bartelmann, HEALPix: A framework for high-resolution discretization and fast analysis of data distributed on the sphere, Astrophys. J. 622, 759 (2005).

[49] A. Zonca, L. Singer, D. Lenz, M. Reinecke, C. Rosset, E. Hivon, and K. Gorski, healpy: Equal area pixelization and spherical harmonics transforms for data on the sphere in Python, J. Open Source Software 4, 1298 (2019).

[50] E. M. George, C. L. Reichardt, K. A. Aird et al., A measurement of secondary cosmic microwave background anisotropies from the 2500-Square-degree SPT-SZ survey, Astrophys. J. 799, 177 (2015).

[51] G. De Zotti, R. Ricci, D. Mesa, L. Silva, P. Mazzotta, L. Toffolatti, and J. González-Nuevo, Predictions for highfrequency radio surveys of extragalactic sources, Astropart. Phys. 431, 893 (2005).

[52] M. Béthermin, E. Daddi, G. Magdis, M. T. Sargent, Y. Hezaveh, D. Elbaz, D. Le Borgne, J. Mullaney, M. Pannella, V. Buat, V. Charmandaris, G. Lagache, and D. Scott, A unified empirical model for infrared galaxy counts based on the observed physical evolution of distant galaxies, Astrophys. J. Lett. 757, L23 (2012).

[53] W. B. Everett, L. Zhang, T. M. Crawford et al., Millimeterwave point sources from the 2500 Square Degree SPT-SZ survey: Catalog and population statistics, Astrophys. J. 900, 55 (2020).

[54] R. Keisler, C. L. Reichardt, K. A. Aird et al., A measurement of the damping tail of the cosmic microwave background power spectrum with the south pole telescope, Astrophys. J. 743, 28 (2011).

[55] J. H. P. Wu, A. Balbi, J. Borrill, P. G. Ferreira, S. Hanany, A. H. Jaffe, A. T. Lee, S. Oh, B. Rabii, P. L. Richards, G. F. Smoot, R. Stompor, and C. D. Winant, Asymmetric beams in cosmic microwave background anisotropy experiments, Astrophys. J. Suppl. Ser. 132, 1 (2001).

[56] M. Lueker, C. L. Reichardt, K. K. Schaffer et al., Measurements of secondary cosmic microwave background anisotropies with the south pole telescope, Astrophys. J. 719, 1045 (2010).

[57] A. Lewis and S. Bridle, Cosmological parameters from CMB and other data: A Monte Carlo approach, Phys. Rev. D 66, 103511 (2002).

[58] A. Lewis, A. Challinor, and A. Lasenby, Efficient computation of cosmic microwave background anisotropies in closed Friedmann-Robertson-Walker models, Astrophys. J. 538, 473 (2000).

[59] D. Jeong, J. Chluba, L. Dai, M. Kamionkowski, and X. Wang, Effect of aberration on partial-sky measurements of the cosmic microwave background temperature power spectrum, Phys. Rev. D 89, 023003 (2014).

[60] A. Manzotti, W. Hu, and A. Benoit-Lévy, Super-sample CMB lensing, Phys. Rev. D 90, 023003 (2014).

[61] U. Natale, L. Pagano, M. Lattanzi, M. Migliaccio, L. P. Colombo, A. Gruppuso, P. Natoli, and G. Polenta, A novel CMB polarization likelihood package for large angular scales built from combined WMAP and Planck LFI legacy maps, arXiv:2005.05600.

[62] R. Adam, P. A. R. Ade et al. (Planck Collaboration), Planck intermediate results. XXX. The angular power spectrum of 
polarized dust emission at intermediate and high Galactic latitudes, Astropart. Phys. 586, A133 (2016).

[63] Y. Akrami, M. Ashdown et al. (Planck Collaboration), Planck 2018 results. XI. Polarized dust foregrounds, Astropart. Phys. 641, A11 (2020).

[64] L. M. Mocanu, T. M. Crawford, K. Aylor et al., Consistency of cosmic microwave background temperature measurements in three frequency bands in the 2500-square-degree SPT-SZ survey, J. Cosmol. Astropart. Phys. 07 (2019) 038.

[65] S. Gratton and A. Challinor, Understanding parameter differences between analyses employing nested data subsets, arXiv:1911.07754.

[66] W. L. Freedman, B. F. Madore, D. Hatt, T. J. Hoyt, I.-S. Jang, R. L. Beaton, C. R. Burns, M. G. Lee, A. J. Monson, J. R. Neeley, M. M. Phillips, J. A. Rich, and M. Seibert, The Carnegie-Chicago Hubble program. VIII. An independent determination of the hubble constant based on the tip of the red giant branch, arXiv:1907.05922.

[67] K. C. Wong, S. H. Suyu, G. C. F. Chen et al., H0LiCOW XIII. A $2.4 \%$ measurement of $\mathrm{H}_{0}$ from lensed quasars: $5.3 \sigma$ tension between early and late-Universe probes, Mon. Not. R. Astron. Soc. 498, 1420 (2020).

[68] S. Birrer, A. J. Shajib, A. Galan et al., TDCOSMO IV: Hierarchical time-delay cosmography-joint inference of the Hubble constant and galaxy density profiles, arXiv: 2007.02941.

[69] F. Bianchini, W. L. K. Wu, P. A. R. Ade et al., Constraints on cosmological parameters from the $500 \mathrm{deg}^{2}$ SPTPOL lensing power spectrum, Astrophys. J. 888, 119 (2020).

[70] C. Heymans, T. Tröster, M. Asgari et al., KiDS-1000 Cosmology: Multi-probe weak gravitational lensing and spectroscopic galaxy clustering constraints, arXiv: 2007.15632.

[71] T. M. C. Abbott, F. B. Abdalla et al. (DES Collaboration), Dark energy survey year 1 results: Cosmological constraints from galaxy clustering and weak lensing, arXiv:1708.01530.
[72] S. Bocquet, J. P. Dietrich, T. Schrabback et al., Cluster cosmology constraints from the $2500 \mathrm{deg}^{2}$ SPT-SZ survey: Inclusion of weak gravitational lensing data from magellan and the Hubble Space Telescope, Astrophys. J. 878, 55 (2019).

[73] S. Alam, M. Ata, S. Bailey et al., The clustering of galaxies in the completed SDSS-III Baryon Oscillation Spectroscopic Survey: Cosmological analysis of the DR12 galaxy sample, Mon. Not. R. Astron. Soc. 470, 2617 (2017).

[74] M. Blomqvist, H. du Mas des Bourboux, N. G. Busca et al., Baryon acoustic oscillations from the cross-correlation of Ly $\alpha$ absorption and quasars in eBOSS DR14, Astron. Astrophys. 629, A86 (2019).

[75] E. Calabrese, A. Slosar, A. Melchiorri, G. F. Smoot, and O. Zahn, Cosmic microwave weak lensing data as a test for the dark universe, Phys. Rev. D 77, 123531 (2008).

[76] L. Knox and M. Millea, Hubble constant hunter's guide, Phys. Rev. D 101, 043533 (2020).

[77] R. Pordes et al., The open science grid, J. Phys. Conf. Ser. 78, 012057 (2007).

[78] I. Sfiligoi, D. C. Bradley, B. Holzman, P. Mhashilkar, S. Padhi, and F. Wurthwein, The pilot way to grid resources using glideinWMS, in 2009 WRI World Congress on Computer Science and Information Engineering, Los Angeles, California, US (IEEE, 2009), Vol. 2, pp. 428-432, https://doi.org/10.1109/CSIE.2009.950.

[79] J. D. Hunter, Matplotlib: A 2D graphics environment, Comput. Sci. Eng. 9, 90 (2007).

[80] E. Jones, T. Oliphant, P. Peterson et al., SciPy: Open source scientific tools for Python, http://www.scipy.org/ (2001).

[81] S. van der Walt, S. Colbert, and G. Varoquaux, The NumPy array: A structure for efficient numerical computation, Comput. Sci. Eng. 13, 22 (2011). 